\title{
LA ESTRUCTURA FUNCIONAL DE LAS NOMINALIZACIONES DEVERBALES DE EVENTO Y RESULTADO A PARTIR DE VERBOS DE REALIZACIÓN
}

\author{
Ana Clara Olivera Polakof \\ Universidad de la República \\ Agencia Nacional de Investigación e Innovación \\ anaclarapo@gmail.com
}

RESUMEN

Este trabajo pretende hacer un aporte al análisis de la estructura funcional de las nominalizaciones de evento y resultado a partir de verbos de realización desde la perspectiva de la morfología ditribuida (MD). Parte del entendido de que en cualquiera de estas estructuras debe haber una relación entre la complejidad morfológica y los nudos funcionales que son contenidos en dicha estructura, así como entiende que la existencia de estas nominalizaciones proviene de una relación entre el componente sintáctico y el conocimiento del mundo. Este trabajo tiene en cuenta los desarrollos previos que se han hecho en MD, así como sostiene la idea de que las nominalizaciones deben contener el Sv a partir del cual se forman y que este Sv tendrá distintos rasgos de $\mathrm{v}^{\circ}$ (Harley y Noyer 2000, Harley 2006, entre otros) que permiten reconocer las distintas clases aspectuales existentes y permiten explicar la relación existente entre las nominalizaciones y los verbos a partir de los cuales estas se forman.

PALABRAS CLAVE: nominalizaciones; evento; resultado; morfología distribuida; estructura funcional.

\section{THE FUNCTIONAL STRUCTURE OF ACCOMPLISHMENT EVENT AND RESULT NOMINALIZATIONS}

\section{ABSTRACT}

This paper tries to make a contribution to the analysis of accomplishment deverbal event and result nominalizations from a Distributed Morphology (DM) point of view. It assumes that there must be a relation between morphological complexity and functional projection that is reflected in each of these structures, and it also assumes that the existence of these nominalizations emerges from an undeniable relation between the syntactic component and the world knowledge. This paper accounts for previous developments that were made in the DM framework and supports the idea that nominalizations must contain the vP from which they are formed and that it will contain different flavours of $\mathrm{v}^{\circ}$ (Harley and Noyer 2000, Harley 2000, and others). This will allow us to recognize different aspectual classes and it will allow us to explain the existent relationship between nominalizations and the verb from which they were formed.

KEY WORDS: nominalizations; event; result; distributed morphology; functional structure.

\section{INTRODUCCIÓN}

El estudio de las nominalizaciones, en un marco generativo, empieza a ser tenido en cuenta con el clásico ensayo de Chomsky (1970) "Remarks on 
nominalizations". ${ }^{1}$ En síntesis, Chomsky propone -para analizar este tipo de formaciones- que es posible explicar los gerundios nominalizados del inglés (John's being eager to please) desde una hipótesis transformacionista debido a las similitudes que estos mantienen con los verbos; mientras que considera que es necesario proponer una hipótesis lexicalista para explicar la existencia de nombres derivados (John's eagerness to please) y en menor medida y claridad para explicar las nominalizaciones mixtas ${ }^{2}$ (John's refusing of the offer) que, si bien mantienen la forma gerundiva, se acercan más al comportamiento de los nombres derivados (Chomsky 1970: 215).

Si bien el trabajo de Chomsky fue tomado como base para elaborar la hipótesis lexicalista, Marantz (1998: 13) considera que el argumento que Chomsky da a partir de las nominalizaciones, más que dar lugar a dicha hipótesis, debe ser considerado como "a knock-out blow against the generative lexicon". Marantz propone, como una teoría alternativa que toma como punto de inicio "Remarks on nominalizations", que no existe un lexicón completamente separado de la sintaxis y que las formaciones de nuevas palabras se dan, también, mediante reglas sintácticas. Esta idea es central en la Morfología Distribuida (MD) en la que se predice "that the structure of words [...] is determined by the syntax and not by subcategorization frames carried by each affix" (Halle y Marantz 1993: 113). Para la MD, es necesario asumir que existen raíces sin categoría gramatical a las que luego se le suman nudos funcionales que permiten distinguir entre, por ejemplo, poblar y población. Se puede observar, entonces, que es posible establecer que, además de dar lugar a la postulación, por otros, de la hipótesis lexicalista, Chomsky (1970) también motiva la postulación de que no existe un lexicón completamente separado de la sintaxis y que las formaciones de nuevas palabras se dan, también, mediante reglas sintácticas, como propone la MD. Esta teoría amplía el panorama del estudio de las nominalizaciones y hubo varios otros ensayos en los que, si bien no siempre estas eran centrales, se trabajaba con las estructuras deverbales (Harley y Noyer 1998; Alexiadou 1999; Embick 2000; Hale y Keyser 1993; entre otros).

La teoría de la MD permite predecir que no son los afijos los que acarrean con las distintas categorías gramaticales, sino que es la sintaxis la que predice cuál será la categoría gramatical de la palabra, como mencionamos (Halle y Marantz 1993:113). Hay tres postulados de este marco teórico que son esenciales

\footnotetext{
${ }^{1}$ Si bien puede ser considerado el marco a partir del cual se estudiaron las nominalizaciones del inglés, debe aclararse que hubo estudios anteriores al de Chomsky que se centraron en las nominalizaciones, como el de Lees (1960) y Vendler (1968), quien toma, como base, al trabajo antes mencionado de Lees.

${ }^{2}$ Sobre este tipo de nominalizaciones y desde la Morfología Distribuida, trabajan Harley y Noyer (1998), quienes mantienen el término propuesto por Chomsky (1970) de mixed nominalizations.
} 
para el estudio de las nominalizaciones. El primero establece que los morfemas son entidades independientes que ocupan nudos funcionales dentro de la estructura jerárquica. El segundo establece que los nudos sintácticos terminales están completamente especificados para el contenido categorial y semántico. El tercero establece que los ítems del Vocabulario deben estar subespecificados y compiten por la inserción de un nudo principal a través del Elsewhere Principle (cf. Harley 2006). Esto implica que el significado no viene determinado desde el lexicón, sino que es posible, a partir de distintas operaciones sintácticas, ir cambiando el contenido y la categoría gramatical de la nueva palabra que se está formando. A su vez, es necesario tener en cuenta que en las nominalizaciones, así como en la formación de otras clases de palabras, va a haber solo dos tipos de morfemas: las raíces que no tienen contenido gramatical pero sí contenido semántico y los elementos gramaticales o morfemas abstractos que asignan la categoría gramatical.

La hipótesis central de nuestro trabajo es que las nominalizaciones, tanto las de evento como las de resultado, poseen una estructura funcional en la que la presencia del nudo funcional verbal categorizador es obligatoria. Si bien se ha probado que las nominalizaciones de resultado se comportan como los nombres comunes contables y tienen rasgos compartidos con ese tipo de nombres (cf. Harley 2006 y Resnik 2010), consideramos que esto no implica que no posean en su estructura funcional un sintagma verbal (Sv). ${ }^{3}$ Esta propuesta tiene en cuenta la tesis de que si hay complejidad morfológica debe haber complejidad en la estructura funcional (cf. Harley 2006). Es decir, si las nominalizaciones de resultado tienen los mismos morfemas que las nominalizaciones de evento, deben tener una estructura funcional que pueda dar cuenta de dicha similitud.

El análisis de la estructura funcional de las nominalizaciones realizacionales de evento y de resultado se hace, entonces, desde la perspectiva de la MD. Parte del entendido previamente enunciado de que en cualquiera de estas estructuras debe haber una relación entre la complejidad morfológica y los nudos funcionales que son contenidos en la estructura, así como entiende que la existencia de estas nominalizaciones puede ser vista como un reflejo de la relación existente entre el componente sintáctico, el sistema conceptual y, en última instancia, el conocimiento del mundo: "In some very intuitive sense, verbs describe events in the world and verbal arguments name individuals that stand in some relevant relations to these events" (Pylkkanen 2002: 10). Las propuestas de estructuras funcionales que hacemos tienen en cuenta los desarrollos previos que se han hecho en MD y muestran que ambas nominalizaciones deben contener el Sv a partir del cual se forman que tendrá como $\mathrm{v}^{\circ}$ distintos rasgos de $\mathrm{v}$ (Harley y Noyer 2000, Harley 2006, entre otros)

\footnotetext{
${ }^{3}$ Entendemos por sintagma verbal un constituyente sintáctico que implica una proyección que "se articula en torno a un verbo" (Bosque y Rexach 2009: 137).
} 
que nos permiten, a su vez, reconocer las distintas clases aspectuales de los verbos a partir de los cuales se forman.

Este trabajo está organizado de la siguiente manera: en primer lugar hacemos una revisión del tratamiento de las nominalizaciones en la lingüística generativa y nos centramos en las propuestas de Grimshaw (1990), Alexiadou (2001), Resnik (2010) y Harley (2006); en segundo lugar, trabajamos con la caracterización de las nominalizaciones deverbales de evento -siempre en el marco generativo- y detallamos las características de estas nominalizaciones en el español; en tercer lugar, hacemos lo mismo pero para las nominalizaciones deverbales de resultado; en cuarto lugar, discutimos los datos analizados y proponemos una estructura funcional para los dos tipos de nominalizaciones deverbales; en quinto lugar, hacemos una síntesis de lo tratado en el artículo y apuntamos algunas conclusiones.

\section{LA NOMINALIZACIÓN EN LA LINGÜÍSTICA GENERATIVA}

Este capítulo muestra la evolución que ha tenido el tratamiento de las nominalizaciones en la lingüística generativa $\mathrm{y}$, si bien se centra en la $\mathrm{MD}$, toma en cuenta el primer tratado completo sobre la estructura argumental que tienen las nominalizaciones que es hecho desde una perspectiva lexicalista. Los trabajos fueron seleccionados debido a que todos ellos proponen estructuras para las nominalizaciones deverbales de evento y de resultado. ${ }^{4}$ Tiene la siguiente organización: en primer lugar mostramos la propuesta lexicalista de Grimshaw (1990); en segundo lugar vemos la propuesta hecha desde la MD de Alexiadou (2001); en tercer lugar se encuentra la propuesta de Resnik (2010) quien reformula algunos de los postulados de Alexiadou para el español; en cuarto lugar trabajamos con la propuesta de Harley (2006) que, consideramos, es la primera propuesta que intenta reanalizar los nombres de resultado. ${ }^{5}$

\subsection{Grimshaw (1990)}

Grimshaw (1990) elabora su propuesta desde la hipótesis lexicalista, más específicamente dentro de la prominence theory. Se puede considerar que este es

\footnotetext{
${ }^{4}$ Si bien podrían haberse tenido en cuenta para los antecedentes otros estudios de la MD como los de Travis (2000), Hale y Keyser (1993), Embick (2000), entre otros, no los consideramos porque no estudian las estructuras funcionales de las nominalizaciones de resultado (algo que sí hacen las investigaciones antes mencionadas y es esencial para nuestro trabajo).

${ }^{5}$ Podrían haberse incluido los trabajos de Osswald (2005) y Sleeman y Brito (2010a y b), entre otros, que analizan la estructura de las nominalizaciones de resultado. El primero fue descartado porque no tiene en cuenta las estructuras de las nominalizaciones de evento y los datos descriptivos son del alemán. El segundo fue descartado porque, si bien se hace desde una perspectiva de la MD, su propuesta se basa en Ramchand (2008) y, por razones que mencionaremos más adelante, consideramos que la propuesta de los rasgos de $\mathrm{v}$ presente en Harley (2006) y otros presenta beneficios respecto a lo que se puede decir de la estructura funcional de los nombres eventivos simples.
} 
el primer trabajo que se centra exclusivamente en la estructura argumental y, más particularmente, en la estructura argumental que tienen algunos nombres. Es, por lo tanto, el punto de partida para un estudio sobre nominalizaciones en MD porque, si no se entendiera la propuesta hecha por Grimshaw, no se podría entender la propuesta de Alexiadou (2001) que veremos en el próximo apartado.

Esta propuesta considera que la estructura argumental (EA) representa "a complex of information critical to the syntactic behavior of a lexical item" (Grimshaw 1990: 1). La estructura argumental es capaz de afectar el comportamiento gramatical del predicado al que pertenece y define la estructura interna del ítem léxico o, más bien, los argumentos que el ítem léxico puede llegar a tener. La EA se construye respetando el principio universal sobre la organización jerárquica de la lengua. En esta jerarquía, según Grimshaw, el agente será siempre el argumento más prominente, al que le seguirán el de experimentante, meta, origen, ubicación y finalmente el tema. ${ }^{6}$

Si bien Grimshaw reconoce la existencia de distintos tipos de nombres (derivados y no derivados), propone que los únicos nombres que pueden poseer estructura argumental (una estructura interna que licencie argumentos) son los que ella llama de evento o proceso complejos. Estos nombres poseen una estructura aspectual que les permite ser visibles para la sintaxis y se podría llegar a plantear que es posible observar la organización temporal interna del evento en ellos (Tenny 1989). Se puede, entonces, dividir los nombres según si tienen una estructura eventiva o no. Dado que los únicos nombres que tienen esta estructura son los nominales eventivos complejos, "the distinction between complex event nominals and the others, then, is a matter not of having temporal extent but of the existence of an internal semantic analysis of the event provided by the event structures [...]" (Grimshaw 1990: 59). Esta distinción tiene repercusiones en la clasificación de los nombres, así como en las representaciones léxicas que los nominales pueden llegar a tener.

La clasificación propuesta por Grimshaw que es resumida, junto con Williams, en 1993, implica la existencia de tres tipos de entidades diferentes que pueden llenar la posición nuclear de sintagmas nominales que son: nombres individuales, eventos simples y eventos complejos:

a) individual: the dog; an exam

b) simple event: the occasion, an event

c) complex event: the examination of the patient; the construction of an example (Grimshaw y Williams 1993: 97)

\footnotetext{
${ }^{6}$ La adaptación al español de los nombres de los papeles temáticos la hicimos siguiendo a Bosque y Gutiérrez-Rexach (2009: 273-274).
} 
Esta clasificación sirve, además de para dividir nombres como los anteriores, para clasificar aquellos nombres que con la misma forma expresan tres lecturas diferentes, como se observa en Alexiadou y Grimshaw (2008: 2):

$\left.a^{\prime}\right)$ The examination of the patients took a long time (Complex)

$b^{\prime}$ ) The examination took a long time (Simple)

$c^{\prime}$ ) The examination was on the table (Result [individual])

Estos ejemplos permiten plantear, además de probar que es posible que una misma forma integre las tres categorías, que es necesario poder dar alguna explicación que logre demostrar cuál es la diferencia estructural que esos nombres tienen que les permite ser clasificados en tres clases que presentan diferentes funcionamientos en la sintaxis y, por lo tanto, distintos significados. Grimshaw propone que la diferencia entre estos tres tipos se debe no solo a su estructura interna, sino que también se debe a los argumentos externos que estos nombres presentan: en el caso de a' tenemos un argumento externo del nombre diferente del que tenemos en los casos de $b^{\prime} y^{\prime} c^{\prime}$. Esta idea refuerza la hipótesis planteada por Grimshaw sobre la existencia de dos grupos de nominales: los de evento complejo y los otros.

Desde una perspectiva semántico-filosófica que parece forzada desde una perspectiva lingüística, esta última propuesta -para la cual la autora sigue a Williams 1981, Di Sciullo y Williams 1987 y Higginbotham 1985-implica que los nombres deverbales tienen los argumentos que heredan del verbo y un argumento externo que es propio de ellos. Este argumento permite diferenciar entre los nominales complejos que presentan un argumento externo no temático Ev que refiere al evento en el mundo y el resto de los nominales (simples e individuales) que presentan un argumento externo no temático $\mathrm{R}$ que les permite referir mediante la adjunción a un determinante. Esta propuesta presenta un rico análisis de los nominales eventivos complejos por lo cual ha sido base para muchos estudios de este tipo de nominalizaciones, pero no tiene en cuenta las diferencias estructurales entre los eventivos simples y los nombres individuales que, a nuestro entender, existen. Por lo tanto, reiteramos, desde esta perspectiva el único análisis posible es aquel que subdivide los nombres eventivos complejos del resto de los nombres y se puede ver en el análisis propuesto por Grimshaw (1990: 68) para los sintagmas nominales:

(1) NP [Det (R) N (R)]

(2) NP [Det (Ev) N (Ev (x (y)))]

\subsection{Alexiadou (2001)}

Alexiadou (2001) es, quizá, el primer intento sistematizado dentro de la Morfología Distribuida de explicar y fundamentar cuál es la proyección funcional de las nominalizaciones en varias lenguas, principalmente el griego y el inglés. Esta perspectiva, en la que las raíces deben unirse a nudos funcionales 
para tener una categoría gramatical determinada, permite explicar las similitudes existentes entre nombres y verbos desde tales proyecciones funcionales.

$\mathrm{Su}$ estudio es más amplio que el de Grimshaw, dado que trabaja no solo con verbos transitivos sino que lo hace con intransitivos, ditransitivos y con predicados psicológicos. A su vez, en contra de la propuesta de Grimshaw, considera que las nominalizaciones, sean de evento o de resultado, poseen estructura argumental y que la diferencia entre estas radica en la proyección funcional que cada uno de estos nombres o nominalizaciones tiene.

Alexiadou (2001: 10) considera que la diferencia entre las nominalizaciones de evento y las de resultado radica en que las primeras incluyen un conjunto de categorías funcionales que se asocian con la lectura de evento o proceso; mientras que las segundas no lo hacen. A su vez, considera que no son solo las nominalizaciones de evento las que poseen complementos sino que también lo hacen las nominalizaciones de resultado, como mencionamos.

Propone que el v "chiquita"7 que hay en las nominalizaciones de evento es el nudo funcional deficiente pues no permite que haya una realización externa del argumento externo. Esto la lleva a afirmar que las nominalizaciones son un reflejo de la generalización de Burzio, ${ }^{8}$ lo que permite explicar algunas de las diferencias entre los verbos y los nombres deverbales. A su vez, permite explicar la diferencia estructural entre las nominalizaciones de evento y resultado que, para Alexiadou, no poseen ninguna proyección verbal. Si bien pueden tomar argumentos, esta posibilidad la tienen porque todos los nombres pueden tomar argumentos. Esto implica que la estructura funcional de las nominalizaciones de resultado que propone es más sencilla que la de las de evento.

Estas diferencias pueden verse en las estructuras que la autora propone para las nominalizaciones de evento (3) y las de resultado (4) (Alexiadou 2001: $19):^{9}$

\footnotetext{
${ }^{7}$ Este término es tomado de la tesis de Resnik (2010: 158), quien utiliza "chiquita" para referirse a "los núcleos funcionales que categorizan a las raíces léxicas".

${ }^{8}$ Esta generalización puede ser expresada como: “Un verbo asigna caso a la posición de objeto (o complemento directo) solo si asigna papel temático a la posición de sujeto" (Bosque y Rexach 2009: 397).

${ }^{9}$ L es la raíz léxica y, por lo tanto, LP sería la frase léxica. Esta frase tiene la particularidad de tener como núcleo la raíz léxica que no tiene una categoría gramatical asignada y sus complementos. Más adelante, utilizaremos la traducción al español $S \sqrt{ }$ que es la sigla para Sintagma Raíz y tiene las mismas propiedades que la que es propuesta por Alexiadou y se encuentra en las estructuras (3) y (4).
} 
(3)

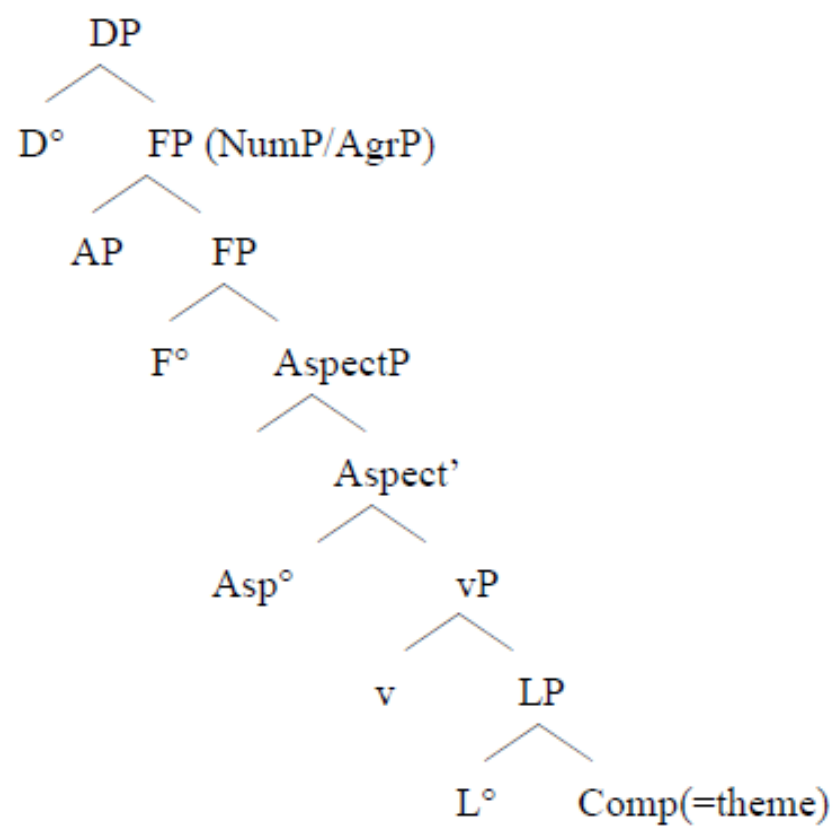

(4)

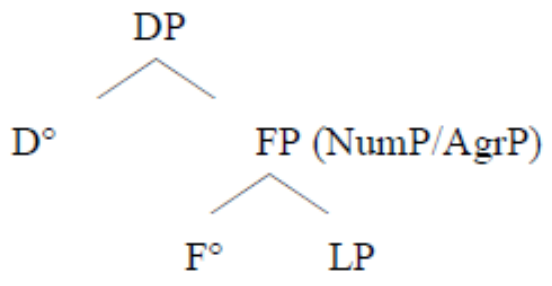

Es posible observar que, en este análisis, hay una semejanza con la propuesta de Grimshaw en el sentido de que la estructura argumental de las nominalizaciones de evento es mucho más compleja que la de las de resultado que comparten, como mencionamos, la estructura con los nombres comunes. La única diferencia, según esta autora, que presentan los nombres de resultado con los nombres comunes es que en los primeros hay un significado de estado resultante en la raíz que los segundos no tienen, pero esto no se ve reflejado en la estructura funcional. ${ }^{10}$ Esto implica, de alguna manera, una simplificación en el tratamiento de la nominalización de resultado que no está presente en la nominalización de evento que coincide con la simplificación de las mismas nominalizaciones en Grimshaw (1990).

\subsection{Resnik (2010)}

La propuesta de Resnik (2010) hace una reformulación de los planteos de Alexiadou (2001) para el español. En su trabajo, Resnik propone ampliar el estudio de los nombres de evento a lo que Grimshaw había clasificado como nombres eventivos simples. Es decir, a aquellos nombres que refieren a un evento pero que no tienen una estructura deverbal. Esta tesis, como las

10 Esta idea, que explica semánticamente la lectura de resultado, permite que nos preguntemos si, para que la raíz tuviese este significado, no sería necesario postular la existencia de una raíz diferente (homófona) en la lista 1 (contiene los morfemas abstactos y las raíces que contienen información semántica). Si esto fuera así, sería necesario hacer una complejización del léxico que podría ser evitada en casos como estos en los que las diferencias de significado pueden explicarse alterando la estructura funcional sin cambiar la raíz a partir de la cual se forman los nombres. 
anteriores, se centra sobre todo en los nominales eventivos aunque hace, también, un tratamiento de las nominalizaciones de resultado que se forman a partir de los mismos verbos que las nominalizaciones eventivas.

Este estudio considera los distintos contextos sintácticos que permiten diferenciar entre los nombres eventivos deverbales, los eventivos no deverbales, los resultativos y los semelfactivos denominales (del tipo de patadas). De esta manera, la autora logra establecer diferencias estructurales entre estos nombres que se pueden ver reflejadas en la estructura funcional. Para establecer estas estructuras, la autora propone que el lugar del argumento externo debe ser similar al del sujeto oracional y, por lo tanto, debe ocupar la posición del especificador del núcleo funcional "que domina el núcleo léxico de la construcción" (Resnik 2010: 144). En las estructuras funcionales que propone, omite los sintagmas funcionales, como el de concordancia, que son externos al sintagma nominal pues no son necesarios para su análisis y es, de acuerdo con la autora, una postura que es generalmente aceptada en los estudiosos de la proyección extendida del nombre (cf. Resnik 2010: 159).

Las estructuras que mostramos en (5) y (6) son adaptaciones al español de la propuesta de Alexiadou (2001) al español, mientras que (7) es la estructura que Rensik (2010: 167) propone para los nombres eventivos no deverbales.

(5)
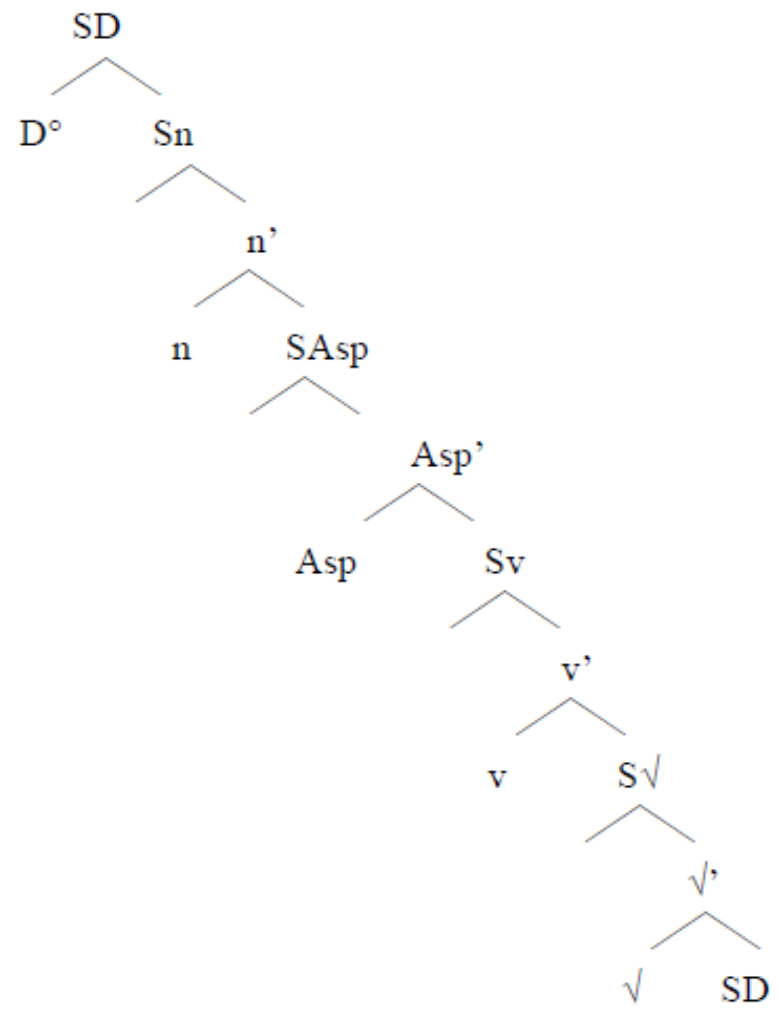

(6)

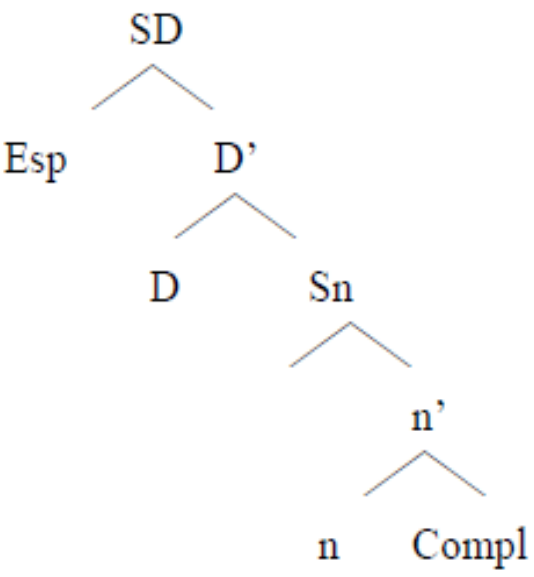


$(7)^{11}$

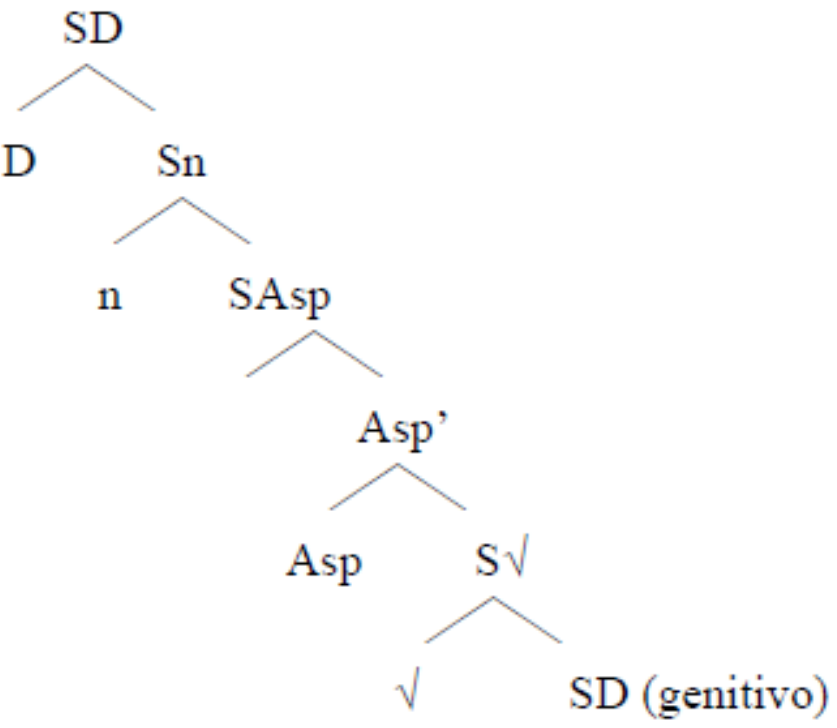

Estas estructuras son formuladas a partir de un estudio detallado de las diferencias sintácticas que expresan los distintos tipos de nombres. Para esto realiza muchas pruebas diagnósticas (cf. Resnik 2010 83-84) que le permiten establecer que las nominalizaciones eventivas y las resultativas están, por ejemplo, en distribución complementaria, mientras que los nombres eventivos simples no. Si bien el estudio de los distintos contextos sintácticos es bien detallado, en lo que respecta a la estructura funcional de estos nombres, el análisis no termina de especificar cuál es la estructura funcional que debemos tener en cuenta.

En páginas previas al análisis propuesto en (6), la autora plantea que en las nominalizaciones a partir de verbos de realización sería posible plantear que, dado que -según autores como Pustejovsky 1991 y Grimshaw 1990- tales verbos poseen dos subeventos (el e 1 que se corresponde con la actividad y el e 2 que se corresponde con el estado resultante), las nominalizaciones eventivas podrían llegar a poseer el e1 y las resultativas el e2. De hecho, afirma que la razón por la cual una variante eventiva puede combinarse con frecuente y una resultativa con sólido se debe, justamente, al hecho de que las nominalizaciones no pueden poseer los dos subeventos del verbo de realización del cual se forman. Sin embargo, más adelante afirma:

De todos modos, el hecho de que los adjetivos de resultado aparezcan también con cualquier nombre no deverbal sin interpretación eventiva, como pared o sala (una pared sólida, una sala lujosa), muestra que no hay efectos sintácticos específicos de "resultatividad", como sí los hay de eventividad (cf. § 3.4.3), por lo que resulta superflua

11 En la tesis de Resnik los nombres eventivos simples son centrales, pero no en nuestro desarrollo. Por lo tanto, si bien mostramos una de las posibles estructuras funcionales que la autora da para este tipo de nombres debido a la relevancia que tienen en su tesis, no trabajamos de ahora en más con ellas. 
la postulación de una estructura eventiva que corresponda solamente a un resultado. Seguiremos sosteniendo, entonces, que dado que el tipo semántico 'estado final' no se distingue en la sintaxis del tipo 'objeto' (y de hecho a menudo lo denotado es directamente un objeto, como en construcción, edición o filmación), las nominalizaciones resultativas carecen de estructura eventiva (Resnik 2010: 151).

Estas afirmaciones, que pueden parecer confusas aquí, resultan de contradicciones -o aparentes contradicciónes- que se dan a lo largo de su tesis pues en ciertas ocasiones afirma que las nominalizaciones de resultado no deben tener una estructura funcional diferente de la del nombre común y en otras parece afirmar que deben tener una estructura funcional relacionada con el verbo. De la cita anterior, Resnik propone la estructura que ya hemos mostrado en (6). Sin embargo, en el capítulo 5 ella propone una nueva estructura funcional para los nombres deverbales de resultado que solo llega a esbozar. En estas nuevas reformulaciones, Resnik propone, en primer lugar, eliminar el nudo Asp de los nombres eventivos simples y reemplazarlos por rasgos del nudo nominal $[+\mathrm{e}] \mathrm{y}[ \pm \mathrm{d}]^{12}$ lo cual los asimila a los nombres simples no eventivos. En segundo lugar, traslada esta propuesta hacia la estructura funcional de las nominalizaciones eventivas y, también, la de las resultativas. En tercer lugar y en contradicción con lo que había propuesto, sugiere una estructura funcional de las nominalizaciones diferente a la que había propuesto. Esta diferencia radica, evidentemente, en la presencia de los rasgos antes mencionados para ambas nominalizaciones: todos los nudos nominales van a tener los rasgos $[ \pm \mathrm{e}]$ y $[ \pm \mathrm{d}]$ asociados. Además, en el caso de las resultativas propone, sin entrar en profundidad, que hay un nudo funcional verbal pero no uno aspectual, que las diferenciaría de las eventivas. Cambia, entonces, lo siguiente para las estructuras funcionales de las nominalizaciones:

(8) $\sqrt{ }+v+$ Asp $[ \pm d]+n[+e][-d]^{13}$

(9) $\sqrt{ }+v+n[-\mathrm{e}][+\mathrm{d}]$

La autora no desarrolla en detalle las estructuras funcionales (8) y (9), pero creemos que pueden dar lugar a los siguientes diagramas arbóreos que nosotros proponemos para interpretar las estructuras anteriores:

\footnotetext{
${ }^{12}$ El primer rasgo tiene que ver con si los nombres tienen una lectura de evento [+e] o no [-e]. El segundo rasgo tiene que ver con la distinción entre nombres de masa y contables. Los de masa no están delimitados y, por lo tanto, son [-d]. Los contables están delimitados y son [ $+\mathrm{d}]$.

${ }^{13}$ En el caso de las nominalizaciones eventivas, vale la pena aclarar que el [ $\left.\pm \mathrm{d}\right]$ del nudo aspectual tiene que ver con la telicidad y atelicidad y no con el hecho de ser contables o de masa que tiene que ver con los rasgos del nombre, como mencionamos.
} 
$\left(8^{\prime}\right)$
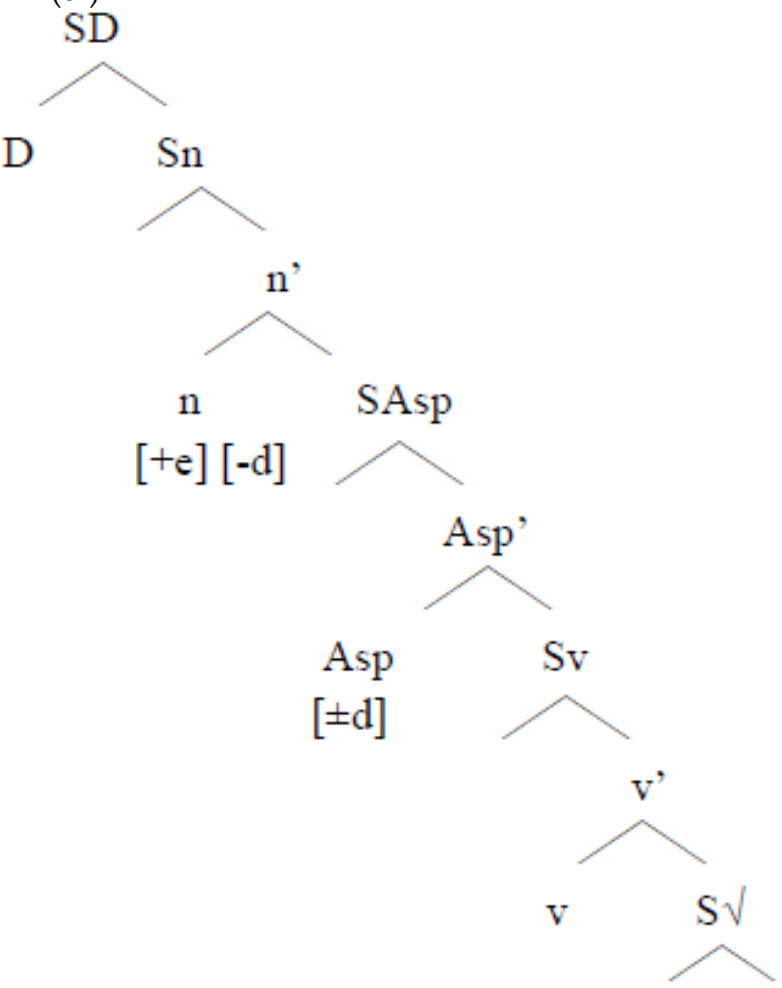

$\left(9^{\prime}\right)$

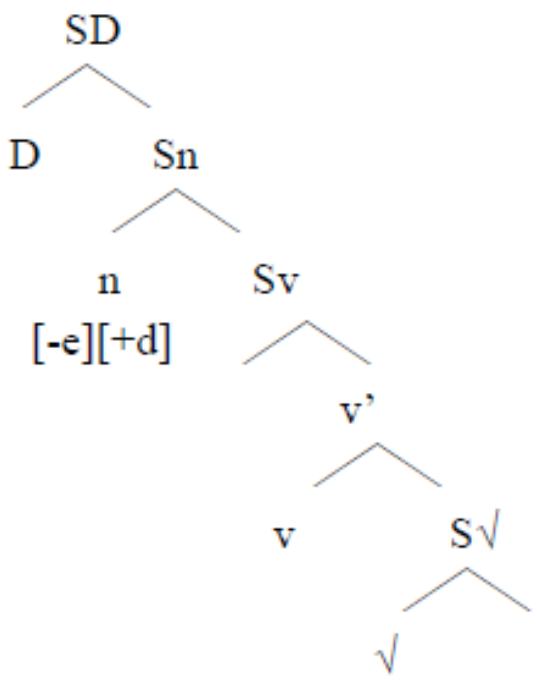

Si bien la estructura funcional ( $\left.8^{\prime}\right)$ no es muy diferente de la propuesta en (6), la estructura (9') sí lo es de la estructura (7) e implica una reacomodación de la teoría. En este último caso, ya no es posible establecer que las nominalizaciones de resultado tienen la misma estructura que los nombres simples y respeta la existencia de estructura derivativa del nombre de resultado deverbal. Sin embargo, no entra en detalle sobre qué es lo que implica este cambio en las estructuras funcionales ni fundamenta en profundidad la razón por la cual propone dicho cambio. A su vez, es importante notar que, en esta teoría, si esta fuese la estructura funcional de la nominalización de resultado sería más sencilla que la de evento. Algo que no sucede en la teoría presentada por Harley (2006) que analizamos a continuación.

\subsection{Harley (2006)}

En el 2006, Harley escribe un artículo en el que se propone, a partir del reconocimiento de que el cambio de la lectura eventiva a la resultativa es bastante productivo y predecible en el inglés, encontrar alguna respuesta al problema de cómo derivar el significado de las nominalizaciones de resultado, en el entendido de que -en el marco de la MD- estos deben contener algún tipo de estructura sintáctica pues comparten con las nominalizaciones eventivas la estructura morfológica. En este sentido, es un avance con respecto de las 
propuestas desarrolladas anteriormente pues parte de la base de que este tipo de nominalizaciones debe tener alguna estructura funcional. ${ }^{14}$

En este artículo propone, como lo había hecho anteriormente (Harley 1999 y Folli y Harley 2002) que los $v^{\circ}$ presentes en la estructura verbal deben tener distintos rasgos que permitan explicar la diferencia aspectual que da como resultado distintos tipos aspectuales de verbos. Esto implica que el locus de la diferencia entre eventivos y estativos, eventos causados o espontáneos, actividades y cambios de estado es $\mathrm{v}^{\circ}$. Propone, entonces, mediante una caracterización de rasgos la siguiente clasificación (Harley 2006: 16):

(10)
a. VCAuse: [+dynamic], [+change of state], [+cause]
b. Vвесоме: [+dynamic], [+change of state], [-cause]
c. VDO: [+dynamic], [-change of state], [-cause]
d. Vве: [-dynamic], [-change of state], [-cause]

Esta clasificación debería poder trasladarse a las nominalizaciones deverbales, pues estas mantienen los afijos verbalizadores como -ize (nominalization). Sin embargo, menciona la autora, es evidente que no comparten todos los caracteres verbales con los verbos: las nominalizaciones mixtas (-ing of) del inglés no aceptan modificadores adverbiales por más que puedan presentar los verbalizadores, como en el caso anterior. Por lo tanto, es necesario proponer alguna reformulación desde la MD que permita dar cuenta de estas diferencias a partir de las modificaciones que los v admitan: "it's perfectly clear that these derived nominals can contain verbalizing $\mathrm{v}^{\circ}$ that accounts for the need for adjectival rather than adverbial modification [...]" Harley (2006: 18).

Esto implica que es necesario reformular qué hace y qué repercusiones tiene la presencia del verbalizador en la estructura funcional. Dado que para estas nominalizaciones no están disponibles ni el argumento externo ni el caso acusativo, no puede ser $\mathrm{v}^{\circ}$ quien licencie el argumento externo ni quien seleccione un núcleo que pueda legitimar caso. Si esto es así, se explica sin problemas por qué las nominalizaciones mixtas (-ing of) tienen tantas diferencias con los verbos: los verbos contienen un $\mathrm{Sv}$ que es dominado por SFlex y Svoz (VoiceP), ${ }^{15}$ que son quienes pueden, efectivamente, asignar caso acusativo y seleccionar argumento externo respectivamente, como se ve en la estructura que propone para los verbos (11); mientras que para las nominalizaciones la estructura (12) no contiene dichas frases, lo que explica por

\footnotetext{
${ }^{14}$ Debemos exceptuar la última parte analizada de Resnik (2010) en la que claramente aparece, al menos, la mención de la necesidad de que las nominalizaciones deverbales de resultado presenten $\mathrm{v}$.

15 El Sflex es un sintagma funcional que tiene como núcleo la flexión verbal y permite seleccionar acusativo. El SVoz (VoiceP de Kratzer 1996) permite seleccionar el agente. El primero tiene como complemento al Sv y el segundo al Sflex, como puede observarse en (11).
} 
qué no pueden este tipo de nominalizaciones seleccionar argumento externo ni asignar caso acusativo y necesitan la presencia de preposiciones como of en el inglés:

$(11)$
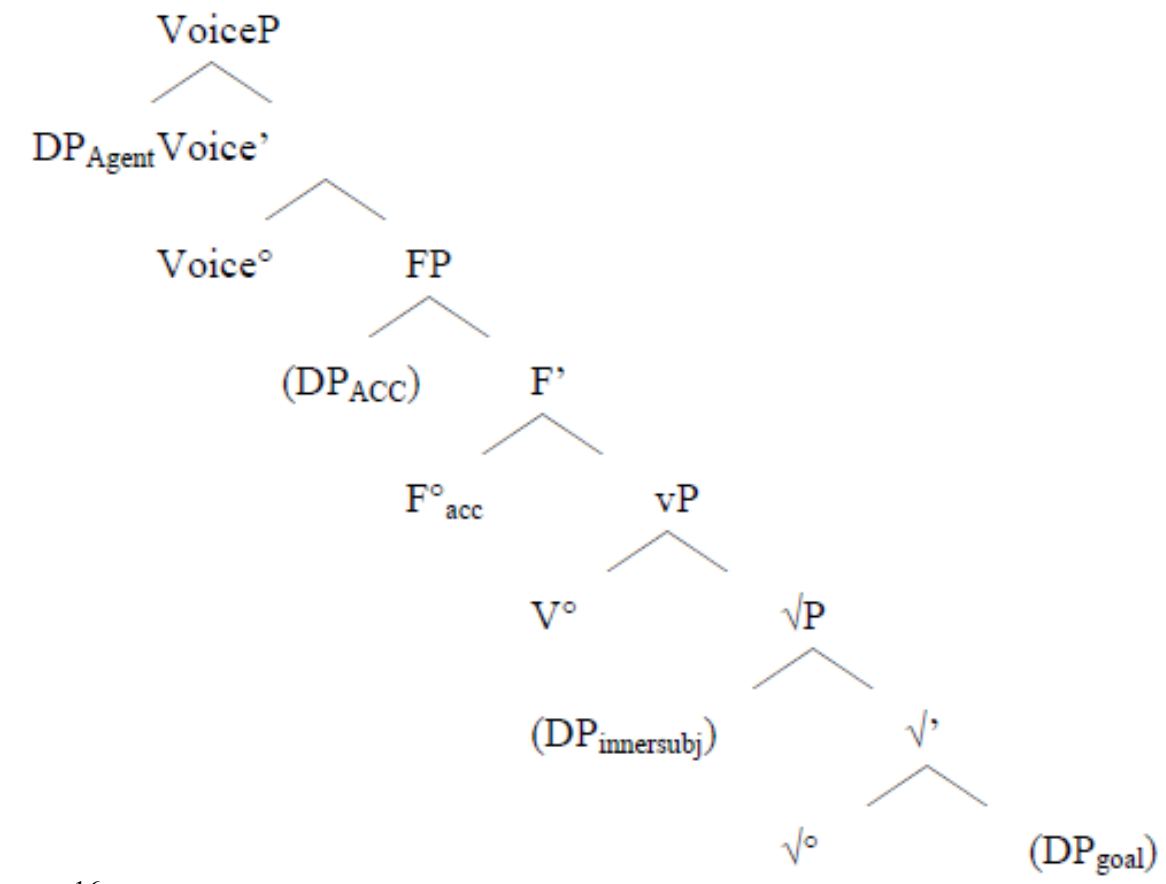

$(12)^{16}$

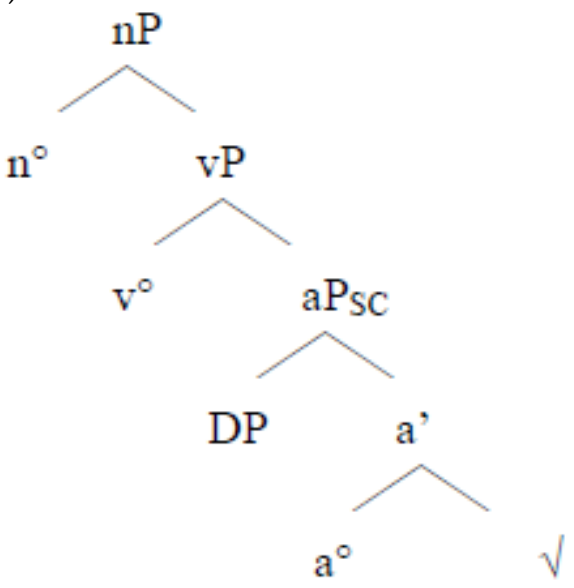

Hasta el momento, la autora ha explicado por qué, si bien comparten parte de la estructura con los verbos, las nominalizaciones no se comportan de la misma manera que los verbos a partir de los cuales se forman. Sin embargo, resta explicar cómo diferenciar entre las nominalizaciones eventivas, como la analizada en (12) de las nominalizaciones de resultado, pues, dado que presentan una morfología compleja, deben tener una estructura funcional

16 Hay que tener en cuenta que, al estar Harley analizando nominalization, es necesaria la frase adjetival que no estaría en el caso de nominalizaciones del tipo de ampliación, por ejemplo. 
compleja. Harley propone que la estructura funcional de las nominalizaciones de resultado, además de ser compleja, debe ser capaz de reflejar el hecho de que, para el inglés no pueden tomar argumentos pues la presencia de estos argumentos fuerza la lectura eventiva. Hay, también, un cambio de interpretación: los nombres eventivos se comportan como los nombres de masa y los resultativos como los contables, es decir que es posible afirmar que estas nominalizaciones se transforman mediante una coerción de una interpretación de masa a una contable como sucede con cualquier nombre. Propone, entonces, que "in undergoing the coercion from a mass, process-denoting nominal to a count, result-denoting nominal, a semantic side-effect kicks in which rules out the presence of the syntactic object" (Harley 2006: 25).

En (12), el objeto (o cláusula reducida que contiene el objeto) es el que se encarga de medir la duración del evento. En el caso, por ejemplo, de que nominalizáramos dos verbos, la nominalización duraría hasta que nominalizáramos ambos verbos y luego finalizaría. Es decir que es el objeto el que provee un límite para el desarrollo del evento, que es en realidad un tema incremental (Dowty 1991). La autora afirma que, en el caso de las nominalizaciones de resultado, algo similar debe ocurrir, i.e. debe haber algo que delimite o marque la lectura contable y que impida la lectura eventiva. Para la autora (Harley 2006: 26), hay un núcleo que crea nombres contables que impone la lectura de resultado previniendo la aparición del tema incremental por lo que no sería el objeto el que delimitaría el resultado, sino ese núcleo. Este debería dominar el sintagma nominal, como podría hacerlo un nudo nulo de número, según la autora. De esta manera, la estructura funcional del sintagma nominal eventivo sería idéntica a la del sintagma que contiene una nominalización de resultado y la diferencia radicaría en sintagmas funcionales externos al sintagma nominal lo que implica una estrcutura funcional de los resultativos más compleja que la de los eventivos.

\section{LAS NOMINALIZACIONES DEVERBALES DE EVENTO}

Como hemos ido mostrando, el tratamiento de las nominalizaciones de evento ha ido evolucionando desde la seminal obra de Chomsky (1970). Podría afirmarse que, al menos en lo que a la contextualización sintáctica refiere, todos los estudios sobre nominalizaciones deverbales de evento en inglés se han basado en el trabajo de Girmshaw (1990). De acuerdo con sus planteos, puede afirmarse que:

(13) Las nominalizaciones de evento complejas:

a. nombran un proceso o evento

b. tienen estructura argumental y toman argumentos

i. The constant assignment of unsolvable problems is to be avoided ${ }^{17}$

\footnotetext{
${ }^{17}$ Los ejemplos son tomados literalmente de Grimshaw (1990: 49-58).
} 
ii. ${ }^{*}$ The constant assignment is to be avoided

c. aceptan modificación aspectual

i. The constant assignment of unsolvable problems

ii. The total destruction of the city in only two days appalled everyone

d. solo pueden estar determinados por artículos definidos en singular y pueden no tener determinate.

i. They observed the/*an/*one/*that assignment of the problem

e. aceptan un controlador del evento

i. The translation of the book (in order) to make it available to a wider readership ${ }^{18}$

La estructura que propone para un sintagma nominal eventivo bien formado es la que vimos en (2) y reiteramos aquí:

(14) NP [Det (Ev) N (Ev (x (y)))]

En el próximo apartado, trasladamos este análisis al comportamiento sintáctico de las nominalizaciones deverbales de evento para el español.

\subsection{Las nominalizaciones deverbales de evento en español}

En este apartado nos centramos en el estudio de Picallo (1999) y en la actualización hecha por Rensik (2010), quien demuestra que hay otros contextos sintácticos que deben ser tenidos en cuenta para caracterizar correctamente las nominalizaciones deverbales de evento en el español.

Si seguimos los planteos de Picallo (1999), es posible establecer que la lectura eventiva de las nominalizaciones se obtiene a partir de los sintagmas nominales pasivos. Estos forman construcciones complejas que tienen como núcleos a nombres deverbales derivados de verbos transitivos ( $\mathrm{y}$, a veces, intransitivos). Los nombres suelen referir a eventos, acontecimientos o procesos "en los que concurre una acción y en los que se interpreta que el tema o paciente es una entidad que queda 'afectada' en algún sentido por el evento que nombra el núcleo" (Picallo 1999: 372).

Se ha dicho, y es lo que mantienen tanto Picallo como Resnik (2010), que las nominalizaciones eventivas presentan una estructura argumental en la que el agente y el tema/paciente deben estar presentes en mayor o menor medida. ${ }^{19}$ Cuando el agente es introducido, debe estar sintácticamente expresado mediante un sintagma preposicional encabezado por la locución preposicional por parte de o la preposición por, como en la ampliación de la oficina por parte de los obreros, que sea complementado por el sintagma nominal del cual dicha

\footnotetext{
${ }^{18}$ Para ver las limitaciones que tiene la propuesta de Grimshaw, tanto para las nominalizaciones de evento como para las de resultado ver Sleeman y Brito (2010a y 2010b).

${ }^{19}$ En mayor o menor medida, en el sentido de lo ya propuesto por Grimshaw (1990): la obligatoriedad de los argumentos en las nominalizaciones no es "tan obligatoria" como en las oraciones. Hay pruebas dadas por Resnik (2010) en las que la ausencia de los argumentos de los nombres eventivos del español no impide la lectura eventiva.
} 
nominalización es núcleo. Debemos tener en cuenta que la expresión sintáctica del agente no es obligatoria en español, aunque siempre se puede recuperar semánticamente, i.e. está semánticamente implícito.

El tema/paciente de las pasivas nominales, de acuerdo con Picallo (1999: 374), siempre debe expresarse sintácticamente, "no sólo para legitimar el elemento agentivo de la construcción sino para propiciar la aparición de cualquier otro argumento que léxicamente admita el núcleo nominal". Este argumento debe ser complemento de un sintagma preposicional encabezado por de. Puede, además, estar expresado mediante un posesivo: su construcción por los obreros. Sin embargo, no puede expresarse mediante un adjetivo de relación (cf. Picallo 1999), aunque puede haber un adjetivo de relación en un sintagma pasivo, siempre que no sea el argumento tema/paciente.

Los nombres de evento, según Grimshaw (seguida por Picallo), no pueden estar determinados ni por adjetivos demostrativos, ni por el artículo indefinido, ni por los cuantificadores llamados débiles. Por esta razón, siempre se encuentran determinados por el artículo definido. Sin embargo, Picallo (1999: 379-380) afirma, contrario a los planteos de Grimshaw, que para el español el artículo definido en plural sí puede determinar algunos nombres de evento siempre y cuando tenga una función aspectual (por ejemplo, en las quemas de los libros por la Inquisición). Esta es otra característica que diferencia las nominalizaciones del inglés de las del español.

A estas caracterizaciones dadas por Picallo (1999), se le pueden sumar algunas de las pruebas sintácticas presentadas por Resnik (2010) que han probado ser útiles en la desambiguación entre las nominalizaciones eventivas y las resultativas. ${ }^{20}$

(15) Pruebas sintácticas para reconocer las nominalizaciones eventivas (Resnik 2010: 80 y sgs.)

a. Cláusulas sustantivas: las nominalizaciones eventivas pueden parafrasearse con una oración subordinada que contenga el verbo a partir del cual se forma la nominalización:

i. La absolución del acusado por parte del juez sorprendió a todos

ii. Que el juez absolviera al acusado sorprendió a todos

b. Pueden combinarse con nombres como hecho o proceso:

i. El proceso de absolución del acusado se llevó a cabo en poco tiempo

c. Pueden retomarse en el discurso con el demostrativo esto:

i. Se mencionó la publicación del trabajo en Nature en 1953 y se señaló que esto/esta significó...

d. Pueden ser argumento interno del verbo presenciar:

i. Los asistentes presenciaron la construcción de la maqueta

e. Pueden ser sujeto de construcciones locativas con ser:

i. La construcción del puente fue durante el verano

\footnotetext{
${ }^{20}$ Para un mayor detalle de estas pruebas, ver Resnik (2010).
} 


\section{LAS NOMINALIZACIONES DEVERBALES DE RESULTADO}

En el caso de las nominalizaciones de resultado, más críticas se le han hecho a Grimshaw (1990). Sin embargo, en Alexiadou y Grimshaw (2008) muestran puntos en común entre las dos perspectivas. Nosotros hacemos una síntesis de los puntos centrales para el planteo de Grimshaw (1990) y luego pasamos a los planteos de Picallo (1999) y Resnik (2010) para el español:

(16) Las nominalizaciones de resultado:

a. Nombran el resultado de un proceso o un elemento asociado con el proceso

b. No tienen estructura argumental y no toman argumentos ${ }^{21}$

i. The assignment is to be avoided

c. No aceptan modificación aspectual

i. *The constant assignment is to be avoided

d. Pueden estar determinados por atículos definidos e indefinidos en singular y en plural, así como otros determinantes

i. They studied the/an/one/that assignment

e. No aceptan un controlador del evento 22

i. ${ }^{*}$ The translations of the book (in order) to make it available to a wider readership

Un sintagma nominal resultativo bien formado debe tener la estructura (1) que volvemos a reiterar:

(17) NP [Det (R) N (R)]

Pasemos ahora al tratamiento de las nominalizaciones deverbales de resultado en español.

\subsection{Las nominalizaciones deverbales de resultado en español}

Si seguimos a Picallo, debemos caracterizar las nominalizaciones de resultado como aquellas que forman sintagmas nominales activos si todos sus

\footnotetext{
${ }^{21}$ Para la autora, pueden tomar participantes que se diferencian de los complementos en que no serían obligatorios. Este ha sido, probablemente, el punto más conflictivo de la caracterización bastante aceptada y divulgada de Grimshaw. De hecho, varios autores han propuesto que las nominalizaciones de resultado sí pueden tener complementos (cf. Alexiadou 2001: 13), que es la postura mayoritariamente aceptada en el marco de la MD.

${ }^{22}$ Se podría llegar a plantear que estas nominalizaciones pueden llegar a tener un controlador del evento, siempre y cuando el evento esté implícito en la construcción. Sleeman y Britos (2010) platean, siguiendo a Britos y Oliveira 1997 (en Sleeman y Britos 2010), que una construcción como A construção do campo de jogos para entreter as crianças trouxe benefícios para a comunidade expresa una lectura resultativa con controlador del evento. De todas maneras, esta perpectiva difiere de la nuestra porque, para nosotros, esa construcción claramente depende de que exista una culminación lógica del evento. Consideramos que, en este caso, la nominalización no expresa un resultado sino que demuestra que las nominalizaciones de evento pueden ser delimitadas o no delimitadas, como muestran los ejemplos que damos en (23).
} 
argumentos están expresados sintácticamente (por ejemplo: la decisión de ir al cine de María). Sin embargo, todos los argumentos de este tipo de nombres presentan una expresión sintáctica optativa. Es importante tener en cuenta que, de acuerdo con la autora, más allá de que sus argumentos estén expresados o no, el referente 'nocional' no cambia. Estos nombres tienen una lectura referencial que ayuda a que dicho referente no cambie: sea un objeto o el resultado de la acción.

El agente de este tipo de sintagmas, cuando se expresa sintácticamente, se introduce mediante un sintagma preposicional encabezado por de (como se puede observar en: la decisión de ir al cine de María). En el caso de las nominalizaciones resultativas, a diferencia de las eventivas, es posible expresar el agente mediante un posesivo, como en su decisión de ir al cine.

El tema/paciente debe ser introducido a su vez por un sintagma preposicional encabezado por de del que es complemento o por un posesivo -si el agente activo no está expresado sintácticamente. Cuando el tema es introducido por un posesivo provoca una lectura ambigua, respecto a la eventiva, que debe ser desambiguada contextualmente (cf. Picallo 1999: 386). Finalmente, el tema de este tipo de sintagmas también puede ser expresado mediante un adjetivo relacional -a diferencia de los eventivos, como puede verse en la producción lechera de los estancieros. Es posible observar, en un caso como el anterior, que lechera solo puede obtener la función temática de tema (podríamos parafrasearlo con de leche para confirmar que tiene la función de tema).

Las nominalizaciones de resultado pueden estar dominadas por cualquier tipo de determinante, pues no presentan restricciones a la hora de la determinación -como ya había mostrado Grimshaw. Los ejemplos que dimos anteriormente se podrían haber expresado como aquella decisión de ir al baile de María, o una producción lechera de los estancieros, sin crear problemas en la interpretación del resultado ni del objeto nocional que sigue siendo siempre el mismo.

Como hicimos para el caso de los nombres deverbales con lectura eventiva, podemos - para ayudar a la desambiguación- agregar algunas de las pruebas sintácticas presentadas por Resnik (2010).

(18) Pruebas sintácticas para reconocer las nominalizaciones resultativas (Resnik 2010: 80 y sgs.)

a. Cláusulas sustantivas: las nominalizaciones resultativas no pueden parafrasearse con una oración subordinada que contenga el verbo a partir del cual se forma la nominalización:

i. La absolución del juez está bien escrita

ii. *Que el juez [lo] absolviera está bien escrito

b. No pueden combinarse con nombres como hecho o proceso:

i. *El proceso de absolución está bien escrito

c. No pueden retomarse en el discurso con el demostrativo esto:

i. La absolución está muy bien escrita. ${ }^{*}$ Esto/esta presenta ordenadamente... 
d. Pueden ser argumento interno del verbo presenciar:

i. *Los asistentes presenciaron la construcción de papel

e. Pueden ser sujeto de construcciones locativas con estar:

i. La evaluación está sobre la mesa

\section{HIPÓTESIS Y DISCUSIÓN DE LA ESTRUCTURA FUNCIONAL DE LAS NOMINALIZACIONES DEVERBALES}

Antes de considerar individualmente las estructuras funcionales de los dos tipos de nominalizaciones deverbales, nos gustaría hacer algunas aclaraciones que parecen ser pertinentes. Estas consideraciones, algunas de las cuales han sido tenidas en cuenta en algunos estudios recientes (Scher 2006, Carrió 2009 y Medeiros 2010), son relevantes para proponer una estructura funcional compleja en cualquiera de los dos tipos de nominalizaciones que estamos revisando.

En primer lugar, consideramos que -siguiendo a Arad (2003)- tanto las nominalizaciones de evento como las de resultado provienen de una raíz que ya se ha unido a un morfema abstracto verbal que crea palabras. Esto se debe a que ambos tipos de nombres tienen una similitud de significado que es innegable. Por lo tanto, proponer, como lo hicieron Grimshaw (1990), Alexiadou (2001), Borer (2003) y Resnik (al menos en parte de su tesis), que los nombres de resultado no tienen estructura argumental o funcional verbal (dependiendo de la perspectiva de la que partan las autoras) contradice la propuesta empíricamente justificada de Arad y tampoco tiene en cuenta que la complejidad morfológica debe verse reflejada en la complejidad estructural. Si estos nombres tienen un significado tan similar que la única ambigüedad léxica proviene de si posee una lectura de proceso o de resultado del proceso -alguno de los subeventos de un evento complejo- no pueden, en ninguno de los dos casos, formarse directamente de la raíz. Este hecho, además de tener consecuencias en la estructura funcional, se deriva del principio de localidad que Arad (2003: 747) reformula a partir de Marantz (2000) de la siguiente manera:

(19) Locality constraint on the interpretation of roots: roots are assigned an interpretation in the environment of the first category-assigning head with which they are merged. Once this interpretation is assigned, it is carried throughout the derivation (Arad 2003: 747).

En segundo lugar, la estructura funcional que proponemos debe evidenciar que los nombres proceden de verbos típicamente realizacionales (accomplishments [Vendler 1967]). Estos son verbos transitivos que pueden ser clasificados como transitivos nucleares pues tienen una caracterización semántica clara dentro del molde 'el agente actúa sobre y causa un efecto en el paciente' (Levin 1999: 2). Estos verbos han sido objeto de varios estudios dentro de la lingüística formal, así como han provocado gran parte de la discusión sobre la 
estructura eventiva y aspectual (cf. Pustejovsky 1991, Dowty 1991, Levin 1999, Higginbotham 2000, entre otros). Consideramos -siguiendo a Pustejovsky 1991 y Levin 1999- que este tipo de verbos está compuesto por dos subeventos: el evento causante o la actividad y el estado resultante. Levin (1999: 9) propone para los eventos complejos la siguiente estructura que es importante para entender a qué se refieren los lexicalistas cuando hablan de un evento complejo: ${ }^{23}$

\section{(20) [ [ $\mathrm{x}$ ACT $<$ MANNER $>$ CAUSE [ BECOME $[\mathrm{y}<$ STATE $>]$ ] ]}

Se podría considerar que, en el caso de los verbos que nos interesan esta estructura léxico conceptual podría servir como marco para construir las nominalizaciones que vamos a analizar. Si bien es claro que, en el caso de los verbos de realización, esta es una estructura posible nos gustaría hacer una diferencia entre aquellos verbos en los que efectivamente hay un estado resultante (Juan rompió el vidrio, en el que el estado resultante es el hecho de que el vidrio se haya roto) y aquellos verbos en los que hay un objeto que resulta (Juan traduce la Biblia, en la que tenemos un objeto resultado que es una traducción de Juan de la Biblia) (ver Osswald 2005). En este trabajo, nos interesan particularmente aquellos verbos que tienen un objeto como resultado final y no aquellos que pueden ser clasificados como estructuras resultativas por lo que la estructura propuesta en (20) no es la estructura que tomaremos en nuestro análisis, lo que nos permite tener en cuenta los planteos considerados a continuación.

En tercer lugar, nos gustaría tener en cuenta algunos de los postulados hechos por Harley (1999), Harley y Noyer (2000), Folli y Harley (2002) y Harley (2006). ${ }^{24}$ Estas propuestas indican la presencia de 'rasgos' de $\mathrm{v}$ chiquita que permiten comprender los distintos comportamientos verbales. Si bien estos trabajos no se centran exactamente en la misma temática, ${ }^{25}$ todos deben tratar con $\mathrm{v}$ pues proponen estructuras funcionales de categorías directamente relcionadas con este. Proponen que, mediante un cambio en el tipo de v que está presente en la estructura, se podría explicar por qué algunos verbos toman un agente o iniciador de la acción en la posición del especificador de la frase

\footnotetext{
${ }^{23}$ Debemos tener en cuenta que, si bien nosotros reproducimos la estructura léxico conceptual propuesta por Levin (1999), no utilizamos este tipo de análisis cuando proponemos la estructura funcional de los nombres.

${ }^{24}$ Algunas de las cuestiones que aquí tratamos ya han sido mencionadas en 1.1.4.

${ }^{25}$ Harley 1999 trabaja sobre el aktionsart de verbos denominales (verbos como saddle); Harley y Noyer 2000 trabajan sobre nominalizaciones y explican ciertas agramaticalidades en esas nominalizaciones (\#John's growth of tomatoes); Folli y Harley (2002) trabajan sobre la alternancia causativo/agentivo en inglés y en italiano (del tipo a. Mario comió una torta / b. La marea se comió la playa.) y Harley (2006) trabaja con la estructura funcional de las nominalizaciones deverbales eventivas y resultativas (como vimos anteriormente).
} 
verbal y otros no lo hacen. Es decir, si tengo un verbo como romper que necesita un iniciador, este requerimiento se puede explicar porque la raíz $\sqrt{ }$ romp- se une a un $\mathrm{Sv}$ que tienen como nudo un $\mathrm{v}^{\circ}$ [CAUSE] que requiere la presencia de un iniciador o causante de la acción. Mientras que un verbo como crecer selecciona un objeto afectado porque la raíz se une a un $\mathrm{Sv}$ que tiene como nudo un $\mathrm{v}^{\circ}$ [BECOME]. Se pueden reconocer, a partir de los artículos antes citados, cuatro tipos

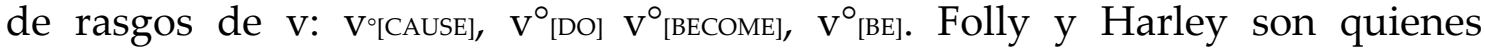

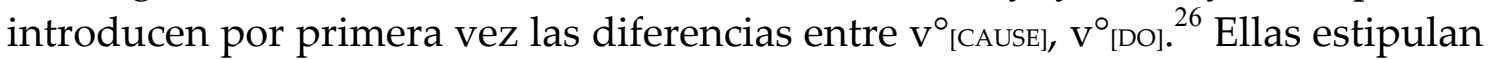
que la diferencia permite explicar que algunos verbos puedan tener como argumento externo una causa (puede ser intencional o no) o que solo puedan tomar un agente intencional y animado. Como vimos, Harley (2006: 16) caracteriza los rasgos de $\mathrm{v}$ de la siguiente manera (que volvemos a citar como 21):

(21)
a. VCAUSE: [+dynamic], [+change of state], [+cause]
b. Vвесоме: [+dynamic], [+change of state], [-cause]
c. VDo: [+dynamic], [-change of state], [-cause]
d. Vве: [-dynamic], [-change of state], [-cause ${ }^{27}$

Carrió (2009) retoma estos planteos para el mocovi y propone una estructura funcional para las nominalizaciones que contiene, en el caso de los eventivos un $\mathrm{v}_{[\mathrm{Do}]} \mathrm{y}$ en el caso de los resultativos un v[BECOME] $\mathrm{y}$ un $\mathrm{v}_{[\mathrm{DO}]}$ que licencia la presencia de un agente obligatorio para las resultativas del mocovi. Nosotros retomamos esta idea porque consideramos que, así como puede explicar las diferencias en el aspecto léxico de los verbos, puede llegar a explicar la diferencia aspectual en las nominalizaciones deverbales. Esto implica que nuestro estudio se podría extender a todo tipo de nominalizaciones teniendo en cuenta estos distintos rasgos de $\mathrm{v}$ que permitirían explicar no solo diferencias aspectuales, sino que -como Folli y Harley (2002) mostraron- permitirían explicar diferencias en la estructura funcional. A su vez, si tenemos en cuenta la consideración de las realizaciones como eventos compuestos por subeventos, podríamos proponer una estructura funcional que -al contener al menos dos rasgos de $\mathrm{v}$ - refleje la complejidad del evento denotado por una realización. ${ }^{28} \mathrm{Y}$

\footnotetext{
${ }^{26}$ Esta propuesta está basada en Hale y Keyser (1993).

${ }^{27}$ Elegimos trabajar con los rasgos de v y no con una propuesta como la de Ramchand (2008) que es seguida por Sleeman y Brito (2010a y b) y por Fabregás (2010) porque, a diferencia de los sintagmas que expresan una lectura aspectual tradicional (ingreso, proceso, resultado), en el caso de los rasgos de $\mathrm{v}$ consideramos que es posible, en concordancia con los planteos de Bohrn (en elaboración), que dichos rasgos se trasladen a los nominales eventivos simples como examen. Esto permitiría explicar sin recurrir a una semántica de la raíz por qué es posible que los nombres tengan lecturas eventivas: en este caso al ser un nombre de realización, podría llegar a presentar los rasgos [DO] y [BECOME] como las nominalizaciones de realización.

${ }^{28}$ Algo similar a lo que propone Travis (2000) -quien reformula la propuesta de Hale y Keyser (1993) con datos del malagasy y talagog- para los SV.
} 
luego, podríamos trasladar este análisis a las nominalizaciones que las resultativas tienen una estructura funcional diferente de la de las eventivas que también debe ser compleja (como proponen Harley 2006, Carrió 2009, entre otros).

En cuarto lugar, nos gustaría aclarar que la estructura funcional que proponemos llega hasta el Sn y no hasta el SD. Esto se debe a que consideramos que las diferencias estructurales entre los nombres deverbales de evento y de resultado -más allá de que haya propuestas de autores como Harley (2006) que predicen que la diferencia es externa al $\mathrm{Sn}$ - se deben a los nudos funcionales con los que se combinan en la interna del Sn. Creemos que en los nudos funcionales que dominan Sn, como Snúm, se incluirá la información gramatical pertinente que haga que en la sintaxis dichas nominalizaciones sean interpretadas correctamente pero no serán quienes determinen cuál lectura es la correcta. Por lo tanto, en el caso de los eventivos, solo será posible la aparición del artículo determinado en singular, mientras que en los resultativos la realización de los nudos funcionales externos estará condicionada por el entorno sintáctico en el que aparezcan, pero estas diferencias no serán tratadas en este momento pues, para nosotros son -en todo caso- reflejo de la estructura funcional del Sn y de las restricciones que este impone; por lo que pueden ser trabajadas en un futuro.

En último lugar, debemos aclarar que la propuesta que nosotros hacemos, tanto para la estructura funcional de las nominalizaciones eventivas como de las resultativas, toma de los antecedentes varias de las bases en las que está asentada. Dentro de estas, tuvimos en cuenta las afirmaciones hechas en (22).

(22)

1. ambos tipos de nominalizaciones, y no solo las de resultado, deben tener una estructura verbal contenida en ellos (como Harley [2006: 2]);

2. los rasgos de $\mathrm{v}$ deben estar presentes en el Sv de la nominalización (como propuso Carrió [2009]);

3. el SAsp debe estar presente en las nominalizaciones de evento (cf. Alexiadou [2001] y Resnik [2010]);

4. las nominalizaciones deben estar contenidas en el Sn, como propusieron Harley (2006) y Resnik (2010), y no en el SD, como propusieron Alexiadou (2001), Carrió (2009), entre otros.

\subsection{Estructura funcional de las nominalizaciones deverbales de evento}

Las cuatro afirmaciones hechas en el apartado anterior deben ser tenidas en cuenta a la hora de proponer la estructura funcional de las nominalizaciones deverbales. Esta estructura debe reflejar que estas nominalizaciones se pueden modificar aspectualmente (como demostraron Grimshaw [1990], Alexiadou [2001], Fu, Roeper y Borer [2001], Resnik [2010], entre otros) y que pueden estar aspectualmente delimitadas o no delimitadas. Los ejemplos de (23) muestran que la presencia del SAsp que aquí defendemos en la estructura funcional de estas nominalizaciones a partir de verbos de realización permite explicar por 
qué es posible construir oraciones delimitadas y no delimitadas. En los caso de a y c tenemos lecturas delimitadas, mientras que en $\mathrm{b}$ y d podríamos plantear que estamos frente a estructuras no delimitadas: ${ }^{29}$

(23)

a. La traducción de la Biblia por parte de los alumnos ha finalizado.

b. La traducción de la Biblia por parte de los alumnos no ha finalizado.

c. La traducción por parte de los estudiantes del texto en dos horas fue lenta.

d. La frecuente traducción de textos por parte de los estudiantes es normal.

Es necesario considerar que estas nominalizaciones, además de tener el SAsp, tienen el Sv que permite explicar que, además de presentar características sobre la telicidad, presenten información funcional proveniente del verbo. Sin embargo, nunca se van a combinar con T. Esto permitiría explicar por qué no es posible, por ejemplo, combinarlas con adverbios de tiempo como ayer ${ }^{*} \mathrm{La}$ traducción ayer de la Biblia por parte de los alumnos) pero sí con adjetivos como frecuente (como se vio en d). Embick (2000: 216-217) es quien propone que las formaciones deverbales contienen los dos sintagmas antes mencionados, pero no T. Plantea que la estructura funcional de dichas formaciones debe tener cierta complejidad estructural que permita explicar por qué tales formaciones tienen implicadas ciertas propiedades aspectuales.

La estructura funcional que proponemos para las nominalizaciones de evento que se muestra en (23) es relativamente más compleja que las que revisamos en 1.1, pero consideramos que mantiene el espíritu de la MD y que permite mantener una relación directa entre el nombre que se forma y el verbo a partir del cual se forma. Es consistente con todos los postulados que plantean que estas nominalizaciones deben tener estructura verbal y se agrega la distinción hecha por Harley (1999), Harley y Noyer (2000), de Folli y Harley (2002) y Harley (2006) sobre los rasgos de v. ${ }^{30}$ Consideramos que la presencia de

\footnotetext{
${ }^{29}$ Alguien podría objetar a esta afirmación el hecho de que lo que dictamina la posibilidad de que la estructura se delimitada o no delimitada es el predicado y no el sujeto. Sin embargo, si cambiáramos la nominalización de evento por un nombre común las oraciones serían agramaticales: *El perro ha finalizado / *El perro no ha finalizado $/{ }^{*}$ El perro en dos horas fue lento / ${ }^{*}$ Los frecuentes perros son normales. Consideramos que esto puede ser tomado como prueba que dictamine la posibilidad de que los nombres de evento sean en sí mismo delimitados o no delimitados.

${ }^{30}$ Si bien la idea de integrar los rasgos de v a la nominalización surge de la tesis de Carrió (2009), no consideramos que la estructura funcional de la nominalización deverbal de evento tenga solo el rasgo Do de v, como propone dicha autora (2009: 115):
}
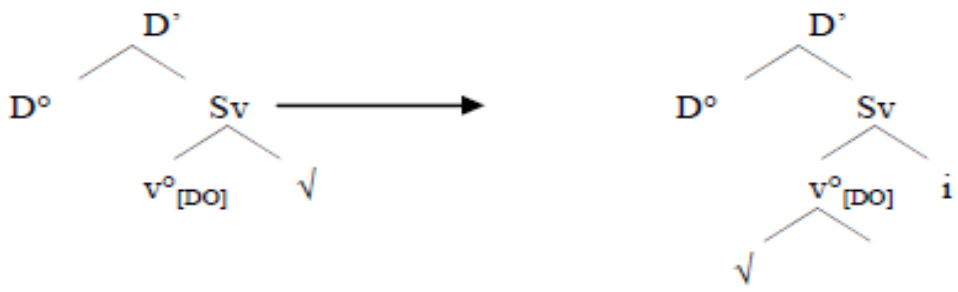
los rasgos de $\mathrm{v}$, no solo permite mantener la relación entre $\mathrm{n} \mathrm{y} \mathrm{v}$, sino que permite explicar por qué no todas las nominalizaciones deverbales se comportan de la misma manera: porque no todos los $\mathrm{v}$ a partir de los cuales surgen tienen los mismos rasgos.

(24) Estructura funcional de las nominalizaciones deverbales de evento a partir de un verbo de realización con un objeto-resultado ${ }^{31}$

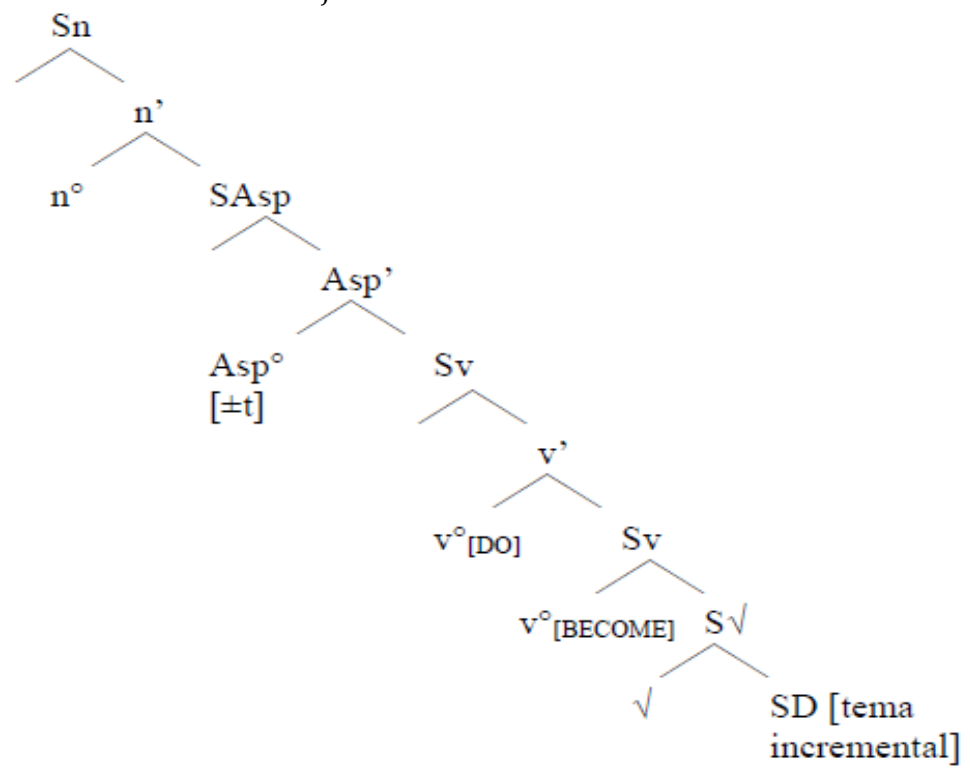

El hecho de que en la nominalización se mantengan los dos rasgos de $\mathrm{v}$ permite explicar, a su vez, por qué es posible que las nominalizaciones deverbales eventivas de realizaciones que tienen como resultado un objeto

Esta idea, si bien parece mantener la noción de proceso, no permite expresar el estado resultante que surge de la combinación con el rasgo BECOME con el tema incremental más su combinación con el predicado que sí es posible en español, como pudo observarse en los ejemplos dados en (23). Si la nominalización de evento solo tuviese el rasgo DO, no podríamos tener una horación como La traducción de la Biblia por Juan ha terminado en la que claramente existe una delimitación del evento que depende no solo del predicado sino de la posibilidad de que la nominalización presente un sintagma aspectual que pueda ser, además de no delimitado (atélico), delimitado (télico)-como hemos mencionado.

Esta estructura es bien diferente de la que nosotros proponemos en (24), no solo porque no

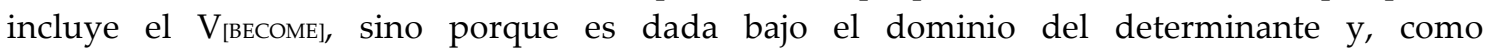
mencionamos, nosotros consideramos que las nominalizaciones deben darse bajo el dominio del Sn pues las diferencias léxicas y funcionales dependen de la estructura interna del Sn.

31 Se podría proponer una estructura funcional, como la que proponen Folli y Ramchand (2005), en la que -en lugar de tener un SD como complemento de la raíz que sea el tema, se tuviera una Frase resultativa que es útil cuando se trabaja con estructuras del tipo John broke the sticks. Sin embargo, como ya mencionamos, debido a que nos interesan aquellas nominalizaciones que provienen de verbos de realización que tienen como resultado un objeto resultado esta parece ser la mejor opción pues permite reflejar que cuando 'alquien hace una traducción de algo obtiene como resultado una traducción'. 
puedan combinarse tanto con palabras como frecuente o construcciones del tipo de en un mes. ${ }^{32}$ Este tipo de modificadores pueden ocupar el lugar del especificador del SAsp dependiendo de si el Asp ${ }^{\circ}$ es [+télico] o [-télico], lo que implica que en el caso de las nominalizaciones eventivas debe ser posible tanto la lectura télica como la no télica. ${ }^{33}$

El rasgo Do de v debe estar por encima de весоме, porque es este rasgo el que puede licenciar un argumento externo como Juan (en la traducción de la Biblia por Juan). El rasgo весоме solo puede tomar como complemento raíces, por lo que debe estar dominado por Do para que pueda aparecer un argumento como Juan (ver Carrió 2009). A su vez, es este rasgo el que nos permite que, en combinación con el tema incremental tengamos la posibilidad de tener una lectura de objeto resultado que no sería posible si tuviéramos a v[DO] en tal posición pues la única lectura posible sería la de proceso atélico o no delimitado. De esta manera, entonces, se explica en combinación con la presencia del SAsp por qué es posible construir oraciones como las que vimos en (23) $\mathrm{y}$, como mencionamos, por la ausencia de $\mathrm{T}$ no es posible hacer combinaciones como *la traducción ayer por Pedro de la Biblia.

Consideramos que la estructura funcional propuesta es capaz de explicar tanto la sintaxis como la semántica de las nominalizaciones eventivas, como hemos mostrado. Sin embargo, hay problemas que no se han tratado que tienen que ver, por ejemplo, con la opcionalidad de los argumentos que podría llegar a explicarse por la falta del SVoz y otros sintagmas funcionales como SFlex. Es decir, si bien los argumentos pueden generarse en el Sv, al no tener categorías funcionales que obliguen a su aparición ni que le asignen caso, estos pueden, a veces, ser omitidos sin perturbar la lectura eventiva (esto se puede seguir de los postulados de Alexiadou 2001).

\footnotetext{
${ }^{32}$ Si bien Resnik (2010: 150-151) reconoce esta realidad, que muestra en los ejemplos (107) b. la frecuente construcción de edificios y (109) La construcción del puente en un mes nos pareció asombrosa, no propone una estructura funcional que pueda dar cuenta de estas diferencias. De hecho, la autora propone (siguiendo a Pustejovsky 1995) que, en casos como (109), "la lectura télica es posible si se introduce una culminación lógica del evento, mediante la realización sintáctica del argumento interno del nombre, pero no es inherente a la nominalización misma". Nosotros consideramos que esto puede explicarse con la presencia del v[BECOME], como hemos mencionado.

${ }^{33}$ Si la lectura es télica, tendremos una formación como la traducción del libro por Juan en dos meses. Si es atélica, podremos formar la traducción frecuente de libros por Juan. Nótese que para la lectura atélica es necesario que el tema esté en plural y, preferentemente, en un sintagma escueto (sin determinante). Lo mismo sucede con la construcción *Juan traducía frecuentemente la Biblia que es agramatical, pero no lo es Juan traducía frecuentemente libros. Esto se debe al hecho de que estamos frente a un tema incremental que ayuda a delimitar el evento, es decir que la telicidad del evento no depende del verbo solo, sino que también lo hace de la estructura en la que esté contenido.
} 


\subsection{Estructura funcional de las nominalizaciones deverbales de resultado}

Si bien la solución dada por Harley (2006) para fundamentar que la estructura funcional de las nominalizaciones deverbales de resultado tenía que ser similar a la de las de evento parecía un poco artificiosa al principio -en el sentido de que postulaba la necesidad de nucleos funcionales externos al Sn que obligaran la lectura resultativa-, parece ser finalmente la postura hacia la cual más nos acercamos. Sin embargo, consideramos que la diferencia debe estar, como mencionamos, en el Sn y no en sintagmas funcionales que estén por encima de este. Por esta razón, proponemos que la diferencia entre las nominalizaciones de resultado y las de evento debe radicar, como han propuesto varios autores, en la ausencia de SAsp. De esta manera, será posible mantener la estructura funcional de Sv y explicar las diferencias en el comportamiento sintáctico entre ambos tipos de nominalizaciones sin recurrir a rasgos del nudo nominal (solución que había propuesto Resnik 2010).

Asumimos que la estructura que proponemos debe poder dar cuenta del hecho de que las nominalizaciones de resultado deben tener una estructura compleja debido a su complejidad morfológica (como propuso Harely 2006). A su vez, entendemos que el hecho de que haya lenguas como el mocovi para las que existe -además del morfema nominalizador eventivo (-agak)- un morfema nominalizador resultativo $(-e k)^{34}$ es una prueba más que nos permite postular tal complejidad estructural. Esto se debe a que, si tenemos en cuenta los planteos hechos por Travis (200: 182), la existencia de una estructura morfológica resultativa para el mocovi, debe ser tenida en cuenta como una confirmación más de que las nominalizaciones de resultado del español deben tener una estructura funcional compleja, ${ }^{35}$ como puede verse en la siguiente cita:

\footnotetext{
${ }^{34}$ Ver Carrió 2009.

${ }^{35}$ Esto no implica que asumamos necesariamente la estructura funcional propuesta por Carrió para las nominalizaciones resultativas del mocovi. Sí implica que la existencia de un morfema diferenciado para expresar el resultado de la acción en una lengua como el mocovi es una prueba de que las nominalizaciones de resultado tienen una estructura funcional compleja que está asociada con el significado resultante de un verbo de realización. Carrió (2009: 116) propone para estas nominalizaciones la siguiente estructura, que es similar a la que proponemos nosotros pero es dominada por un D y no por un Sn, aclaración que hicimos también en el análisis que la autora hace para las nominalizaciones de evento:
}

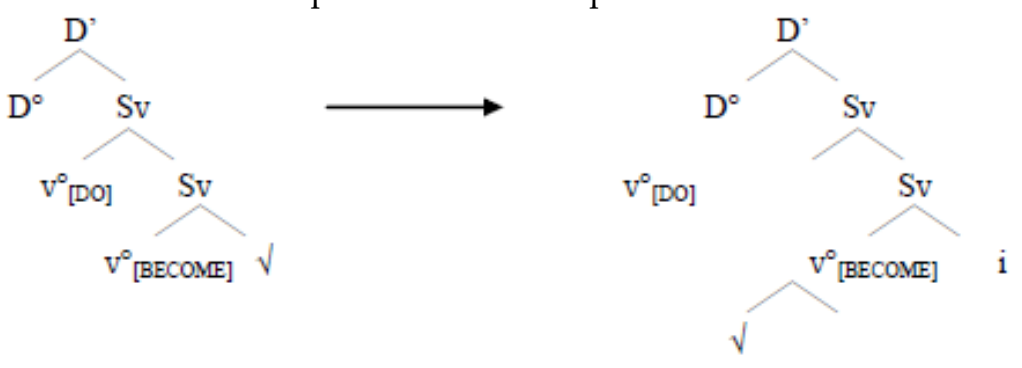


I do assume that if one language has morphological evidence for that head [sintáctica], then all languages will have the same head with the same semantic force even though the morphology may be covert.

(25) Estructura funcional de las nominalizaciones deverbales de resultado a partir de verbos de realización con un objeto resultado

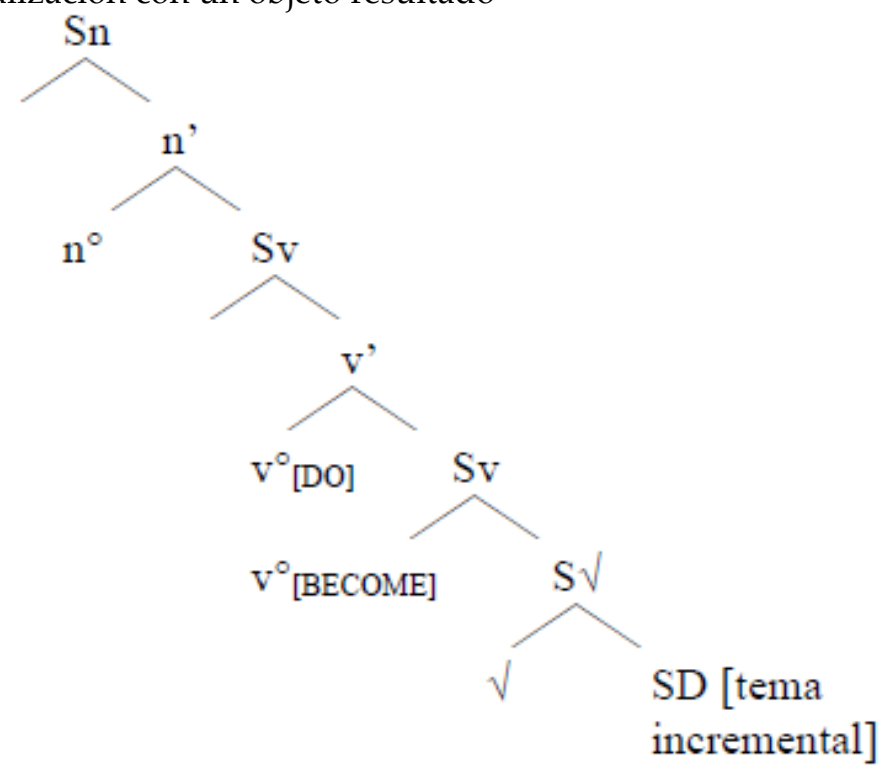

Esta estructura funcional, parecida a la que reformulamos a partir de la tesis de Resnik en (9') y a la propuesta por Carrió (2009) en la nota 36, permite explicar la complejidad morfológica de las nominalizaciones deverbales de resultado, así como permite explicar por qué estas nominalizaciones no tienen una lectura eventiva. Esto se debe, creemos, a la ausencia de SAsp que sería el sintagma encargado de informar sobre la duración del evento o el tipo de duración del evento. Sin esta categoría funcional, el Sv no puede expresar el evento pues el evento, además de tener participantes (en el sentido davidsoniano), debe tener cierta duración en el tiempo. Si bien las nominalizaciones eventivas tampoco tienen $\mathrm{T}$, el hecho de que el SAsp esté presente les permite tener incluida la idea de telicidad o atelicidad -como había propuesto Tenny (1989)-, algo que no pueden tener las nominalizaciones de resultado.

Deberíamos poder explicar por qué es la lectura de resultado la que prima sobre algún otro tipo de lectura, dado que hay dos rasgos de v expresados en la estructura funcional. Esta tarea es, quizá, una de las más complicadas. Creemos que la lectura de proceso -evento- está impedida por la ausencia de SAsp (como mencionamos). Sin embargo, ¿qué impulsa la lectura de resultado? ¿Podemos postular que en la ausencia de SAsp y otros sintagmas funcionales prima el significado de весоме que, es en definitiva, el que nos permite tener un resultado del proceso? Esto parecería ser posible y ya ha sido propuesto por

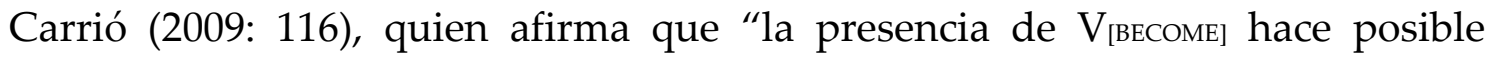
marcar la afectación”. Esta estructura, a su vez, puede explicar por qué es 
posible formar un sintagma como una traducción de Pedro de la Biblia, en la que de Pedro sea interpretado como el agente y no como el poseedor sin necesidad de retirar el Sv de la estructura funcional ni necesidad de proponer que esta estructura debe coincidir con la de los nombres comunes. En todo caso, podríamos afirmar que la ausencia de SAsp y la presencia del Sv de realización permiten establecer que tal Sn pueda ser interpretado como el resultado del proceso que, en el análisis de una nominalización a partir de un verbo como traducir, es un objeto resultado del proceso. ${ }^{36}$

\section{SÍNTESIS Y CONCLUSIONES}

En este trabajo hemos mostrado cuál ha sido la evolución del tratamiento de las nominalizaciones deverbales en la lingüística generativa. Nos hemos posicionado desde la MD en el entendido de que las nominalizaciones son la viva muestra de que la formación de palabras debe darse en la sintaxis y de que esta postura implica una economía de morfemas léxicos que parece sostenerse a partir de los postulados generativos. A su vez, permite demostrar de una mejor forma las relaciones existentes entre las distintas categorías gramaticales y permite explicar, sin la necesidad de recurrir a argumentos externos como Ev o $R$ (como propuso Grimshaw 1990), por qué las nominalizaciones a partir de verbos de realización pueden tener significado eventivo o de objeto resultado.

Hemos visto, a partir de Grimshaw (1990) -con una breve mención a Chomsky (1970)-, que las nominalizaciones han sido centrales para el eje de la discusión sobre la estructura eventiva. Hemos mostrado, cómo, a partir de Alexiadou (2001), se ha intentado en la MD llegar a una estructura funcional de las nominalizaciones que permita explicar no solo el significado eventivo, sino también la estructura funcional que estas nominalizaciones deben tener. Mostramos, también, cuáles son las distintas pruebas sintácticas que nos permiten desambiguar entre la lectura de evento y la de resultado propuestas por Grimshaw y luego las demostradas por Picallo (1999) y Renisk (2010) para las nominalizaciones deverbales en español.

Basándonos en dicho desarrollo, propusimos dos estructuras funcionales diferenciadas para las nominalizaciones deverbales de evento y las de resultado. Ambas comparten la presencia del Sv de realización que implica la

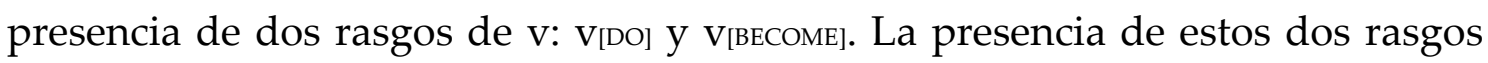
explica la complejidad eventiva que tienen los predicados realizacionales de los

\footnotetext{
${ }^{36}$ Esto se debe al hecho de que, si asumimos la complejidad del evento realizacional y que la nominalización resulta de la unión con un Sv, la nominalización debe tener una lectura relacionada con el significado de la cabeza a la que se une (obligada por el locality constraint expresado en (20)). Por lo tanto, las únicas lecturas posibles para las nominalizaciones deverbales de realización serán las de evento y resultado. Entonces, en el caso de que el SAsp no esté, solo queda habilitada la lectura de resultado.
} 
que provienen, como ya había propuesto Pustejovsky en 1991, así como permite relacionar las nominalizaciones con dichos predicados.

Finalmente, propusimos que la diferencia en la estructura funcional de las nominalizaciones deverbales de evento y resultado no radica en el Sv sino que radica en la ausencia del SAsp. Esto permite explicar por qué las nominalizaciones de resultado no pueden ser modificados por frecuente ni por en un mes, así como el hecho de que no tengan una lectura eventiva pues no tienen el rasgo de telicidad ni el de atelicidad que habilita dicha lectura. Concluimos, entonces, que se puede explicar la lectura de resultado por la ausencia del mismo sintagma (SAsp) y por la lectura de objeto afectado que permite la presencia del rasgo $\mathrm{V}_{[\mathrm{BECOME}]}$ en combinación con el V[DO]; mientras que la presencia de estos mismos elementos, así como la presencia de SAsp, es lo que permite que las nominalizaciones deverbales de evento tengan una lectura eventiva y puedan ser modificadas por distintos tipos de estructura, como frecuente y en un mes.

Nos gustaría concluir este trabajo mencionando que consideramos que es posible trasladar este análisis hacia otro tipo de nominalizaciones y que, mediante el cambio de los rasgos de v y la presencia o ausencia de SAsp, será posible explicar por qué algunas nominalizaciones deverbales tienen lectura solo eventiva, resultativa o estativa. Por lo tanto, sería posible explicar estas diferencias aspectuales en la estructura funcional misma del Sn, sin tener que recurrir a nudos funcionales externos ni a recursos artificiosos como los que ha propuesto, por ejemplo, Grimshaw (1990).

\section{BIBLIOGRAFÍA}

Alexiadou, A. (1999), "Remarks on the Syntax of Process Nominals: An Ergative Pattern in Nominative-Accusative Languages", en Procceedings of NELS 29.

AleXIADOU, A. (2001), Functional structure in nominals: nominalization and ergativitiy, Amsterdam/Philadelphia, John Benjamins Publishing Company.

AleXIADOU, A. y GRIMSHAW, J. (2008), "Verbs, nouns and affixation”, en Working Papers of the SFB 732 Incremental Specification in Context 01, 1-16.

ARAD, M. (2003), "Locality constraints on the interpretation of roots: the case of Hebrew denominal verbs", en Natural Language E Linguistic Theory 21, 737-778.

BOHRN, A. (en elaboración), La herencia morfológica en un marco no lexicalista. Un estudio sobre la transferencia aspectual y argumental en verbos denominales y deadjetivales, Tesis de doctorado, Universidad de Buenos Aires.

BORER, H. (2003), “Exo-skeletal and endo-skeletal explanation: Syntactic projections and the lexicon", en The Nature of Explanation in Linguistic Theory, Moore, J. y Polinsky, M. (eds.), Stanford, CSLI, 31-67.

Bosque, I. y Demonte, V. (dirs.) (1999), Gramática Descriptiva de la Lengua Española, Madrid, Espasa.

BosQue, I. y GUTIÉRREZ-REXACH, J. (2009), Fundamentos de sintaxis formal, Madrid, AKAL. 
CARrIó, C. (2009), Mirada Generativa a la Lengua Mocoví (Familia Guaycurú), Tesis presentada para aspirar al grado de Doctor en Letras, Universidad Nacional de Córdoba.

CHOMSKY, N. (1970), "Remarks on nominalizations", en Readings in English Transformational Grammar, Jacobs, R. y Rosenbaum, P., Waltham, MA, Ginn and Company, 184-221.

DAVIDSON, D. (2001 [1981]), Essays on Actions and Events, New York, Oxford University Press.

Di Sciullo, A. M y Williams, E. (1987), On the Definition of Word, Cambridge, Mass., MIT Press.

DOWTY, D. R. (1991), “Thematic Proto-Roles and Argument Selection”, en Language, 67, 547-619.

EMBICK, D. (2000), "Features, Syntax, and Categories in the Latin Perfect", en Linguistic Inquiry 31, 185-230.

FABREGÁS, A. (2010), "A syntactic account of affix rivalry in Spanish nominalizations", en The Syntax of Nominalizations across Languages and Frameworks, Alexiadou, A. y Rathert, M. (eds.), Berlin/New York, De Muyter Grouton, 67-92.

FolLI, R. y HARLEY, H. (2002), “Consuming Results in Italian and English: Flavours of $\mathrm{v}^{\prime \prime}$, en Aspectual Inquiries, P. Kempchinsky y Slabakova, R. (eds.), Dordrecht, Kluwer, 95-120.

FOLLI, R. y RAMCHAND, G. (2005), "Prepositions and results in Italian and English: an analysis from event decomposition", Perspectives on Aspect, en Verkuyl, H. J. de Swart, H. y van Hout, A. (eds.), Springer, 81-105.

Fu, J., ROEPER, T. y BORER, H. (2001), "The VP within Process Nominals", en Natural Language and Linguistic Theory 19, 549-582.

GrimsHAW, J. (1990), Argument Structure, Cambridge, Massachusetts, London, The MIT Press.

GRIMSHAW, J. y WILLIAMS, E. (1993), “Nominalization and predicative prepositional phrases", en Pustejovsky, J., Semantics and the lexicon, Boston, Kluwer Academic Publishers, 97-106.

HALE, K. y KEYSER, S. J. (1993), “On argument structure and the lexical expression of syntactic relations", The View from Building 20: Essays in Linguistics in Honor of Sylvain Bromberger, en Hale K. y Keyser, S. J. (eds.), Cambridge, Mass., MIT Press, 51-109.

HALle, M. y MARANTZ, A. (1993), “Distributed Morphology and the pieces of inflection", The View from Building 20: Essays in Linguistics in Honor of Sylvain Bromberger, en Hale, K. y Keyser, S. J. (eds.), Cambridge, Mass., MIT Press, 111176.

HARLEY, H. (1999), "Denominal verbs and aktionsart", en MITWPL 35, editado por Heidi Harley.

HARLEY, H. (2006), "The morphology of nominalizations and the syntax of vP", [consulta: 22 marzo 2013]. Disponible en: <http://ling.auf.net/lingbuzz/000434>.

HARLEY, H. y NOYER, R. (1998), “Mixed nominalizations, short verb movement and object shift in English", en Proceedings of NELS 28, 1-15

HARLEY, H. y NOYER R. (2000), “Formal vs. encyclopedic properties of vocabulary: Evidence from nominalizations", en The Lexicon-Encyclopedia Interface, Peeters, B. (ed.), Amsterdam, Elsevier, 349-374. 
HigGinBOTHAM, J. (1985), “On semantics”, en Linguistic Inquiry 16, 547-593.

HigginbothaM, J. (2000), Accomplishments, Manuscript, University of Oxford.

KRATZER, A. (1996), "Severing the external argument from its verb", en Phrase structure and the lexicon, Rooryck, J. y Zarig, L. (eds.), Dordrecht, Kluwer Academic Publishers, 109-137.

LEES, R. (1960), The Grammar of English Nominalizations, The Hague, Mouton.

LEVIN, B. (1999), “Objecthood: an event structure perspective”, en Proceedings of CLS.

MARANTZ, A. (1998), “No Escape From Syntax: Don't Try Morphological Analysis in the Privacy of Your Own Lexicon", en Proceedings of the 1998, Dimitriadis, A. (ed.), Penn Linguistics Colloqium, disponible en Penn Working Papers in Linguistics.

MARANTZ, A. (2000), "Roots: The Universality of Root and Pattern Morphology", trabajo presentado en the conference on Afro-Asiatic languages, University of Paris VII.

MedeIROS, A. (2010), "Aspeto e estrutura de evento nas nominalizaçoes em -ada”, en Revista Letras, N.81, 99-122.

OsswALD, R. (2005), "On result nominalizations in German”, en Proceedings of Sinn und Bedeutung, 9, 256-270.

PICALLO, M. C. (1999), “La estructura del sintagma nominal: las nominalizaciones y otros sustantivos con complementos argumentales", en Gramática Descriptiva de la Lengua Española, Bosque, I. y Demonte, V. (dirs.), Madrid, Espasa, 365-393.

PustejOVSKY, J. (1991), “The syntax of event structure”, en Cognition, 41, 47-81.

PUsteJOVSKY, J. (1995), The Generative Lexicon, Cambridge, Massachusets, MIT Press.

PYLKKANEN, L. (2002), Introducing arguments, PHD dissertation, MIT.

RAMCHAND, G. (2008), Verb Meaning and the Lexicon: A First Phase Syntax, Cambridge, Cambridge University Press.

RESNIK, G. (2010), Los nombres eventivos no deverbales en español, Tesis de doctorado, Barcelona, Universitat Pompeu Fabra.

SCHER, A. P. (2006), “Nominalizações em -ada em Construções com o Verbo Leve dar em Português Brasileiro", en Letras de Hoje, 41 (1), 29-48.

SleEmAN, P. y BRITO, A. M. (2010a), “Nominalization, Event, Aspect, and Argument Structure: a Syntactic approach", en Argument Structure from a Crosslinguistic Perspective, Duguine, M. et al. (eds.), Amsterdam/Philadelphia, John Benjamins, 113-130.

SLEEMAN, P. y BRITO, A. M. (2010b), “Aspect and argument structure of deverbal nominalizations: A split $v \mathrm{P}$ analysis", en The Syntax of Nominalizations across Languages and Frameworks, Alexiadou, A. y Rathert, M. (eds.), Berlin/New York, De Muyter Grouton, 199-218.

TENNY, C. (1989), "The aspectual interface Hypothesis", en Lexicon Project Working Papers 31, Cambridge, MIT.

TRAVIS, L. (2000), "Event structure in syntax", en Events as grammatical objects, Tenny, C. y Pustejovsky, J. (eds.), Stanford, CSLI, 145-185.

VENDler, Z. (1967), "Verbs and times", en Linguistics and Philosophy, Ithaca, NY, Cornell University Press.

VENDLER, Z. (1968), Adjectives and Nominalizations, The Hague, Mouton.

WILLIAMS, E. (1981), "Argument Structure and Morphology", en The Linguistic Review, $1,81-114$. 